\title{
Neuroprotector Effect Of Strength Training In An Neuroinflammation Animal Model
}

Elizama de Gregório ( $\nabla$ elizamagregorio@outlook.com )

Universidade Alto Vale do Rio do Peixe https://orcid.org/0000-0002-9081-9815

\section{Gabriela Cristiane Mendes}

UNIPAMPA: Universidade Federal do Pampa

\section{Lincon Bordignon Somensi}

UNIARP: Universidade Alto Vale do Rio do Peixe

\section{Luiza Freitas Lopes}

UNIPAMPA: Universidade Federal do Pampa

Karine Ramires Lima

UNIPAMPA: Universidade Federal do Pampa

\section{Guilherme Salgado Carrazoni}

UNIPAMPA: Universidade Federal do Pampa

\section{Ben-Hur Souto Neves}

UNIPAMPA: Universidade Federal do Pampa

\section{Steffanie Severo Picua}

UNIPAMPA: Universidade Federal do Pampa

\section{Luísa Mota da Silva}

UNIVALI: Universidade do Vale do Itajai

\section{Pamela Billig Mello Carpes}

UNIPAMPA: Universidade Federal do Pampa

Juliana Sartori Bonini

UNICENTRO: Universidade Estadual do Centro-Oeste

Weber Cláudio da Silva

UNICENTRO: Universidade Estadual do Centro-Oeste

\section{Research}

Keywords: Neuroinflammation, strength training, oxidative stress, memory

Posted Date: August 5th, 2021

DOI: https://doi.org/10.21203/rs.3.rs-764281/v1 
License: (c) (i) This work is licensed under a Creative Commons Attribution 4.0 International License. Read Full License 
1 NEUROPROTECTOR EFFECT OF STRENGTH TRAINING IN AN

2 NEUROINFLAMMATION ANIMAL MODEL

3

4 Elizama de Gregório ${ }^{1,5^{*}}$

5 Gabriela Cristiane Mendes ${ }^{2}$

6 Lincon Bordignon Somensi ${ }^{1}$

7 Luiza Freitas Lopes²

8 Karine Ramires Lima²

9 Guilherme Salgado Carrazoni²

10 Ben-Hur Souto Neves ${ }^{2}$

11 Steffanie Severo Picua²

12 Luisa Mota da Silva ${ }^{3}$

13 Pamela Billig Mello-Carpes ${ }^{2,5}$

14 Juliana Sartori Bonini ${ }^{4}$

15 Weber Cláudio da Silva ${ }^{4,5}$

16

17

181 University of Alto Vale do Rio do Peixe (UNIARP), Department of Medicine,

19 Caçador, SC, Brazil

202 Physiology Research Group, Stress, Memory and Behavior Lab, Federal

21 University of Pampa, Uruguaiana, RS, Brazil

223 Postgraduate Program in Pharmaceutical Sciences (PPGCF), Center for 23 Chemical-Pharmaceutical Research (NIQFAR), University of Vale do Itajaí 24 (UNIVALI), Itajaí, SC, Brazil 
254 Laboratory of Neuropsychopharmacology, Pharmacy, Department, Midwest

26 Stat and University, Guarapuava, PR, Brazil

275 Postgraduate Program in Physiology, Physiology Department, Federal

28 University of Rio Grande do Sul (UFRGS), Porto Alegre, RS, Brazil

$29{ }^{\star}$ Correspondence: elizamagregorio@outlook.com. Department of Medicine,

30 University of Alto Vale do Rio do Peixe (UNIARP), Caçador, SC, Brazil.

32 ABSTRACT

33

34 Background: The preventive role of muscular strength in the diminishing of a 35 neuroinflammation is yet unknown. In this study, the role of the prophylactic 36 muscular strength exercise was investigated, whether it would diminish cognitive 37 alterations and modify the antioxidant intracellular scenery in an animal 38 neuroinflammation model of the CA1 region of the hippocampus. Methods: The 39 animals received muscular strength training for eight weeks, three times a week.

40 Subsequently, the stereotaxic surgery, with intra-hippocampal infusion of either 41 saline solution or lipopolysaccharide (LPS) was performed. Next, behavioral tests 42 were performed: objects and social recognition. At last, the animals were 43 euthanized and the collect of the hippocampus and the prefrontal cortex were 44 performed and, later, the dosage of the antioxidant activity was performed.

45 Results: The results showed that the muscular strength exercises was capable 46 of showing a beneficial prophylactic effect in the oxidative stress caused by an 47 acute neuroinflammation. There was diminishing of the reduced glutathione 48 concentration (GSH) and increase of the activity of the catalase enzyme (CAT) 
49 in the group (SE + LPS), regarding the control groups. In the prefrontal cortex,

50 there was only an increase of the CAT activity in the group (SE + LPS), regarding

51 the groups $(C T)$ and $(S E+S A L)$. As for the cognitive alterations there were found

52 in the (SE + LPS) group, diminishing the mnemonic hazard of the discriminative

53 and social memories, when compared to the control groups. Conclusion: We

54 concluded, therefore, that the induction of a local inflammatory process in the

55 hippocampus leads to mnemonic deficits in behavioral activities and increase of

56 the GSH concentration, and that the muscular strength exercise performed

57 prophylactically presents a protective effect capable of minimizing such

58 mnemonic deficits and increasing the antioxidant defenses in mice that suffered

59 a local neuroinflammatory process in the hippocampus.

60

61 KEYWORDS

62 Neuroinflammation; strength training; oxidative stress; memory.

63

64 ABBREVIATION LIST3

65 AP - antero-posterior

66 BBB - blood-brain barriers

67 BDNF - neurotrophic factor

68 CAT - catalase

69 CCL2 - C-C chemokine ligand 2

70 CNS - central nervous system

71 CO2 - cyclooxygenase 2

72 CT - control group 
73 DTNB - 5,5'-dithio-bis-[2-nitrobenzoic acid

74 DV - dorsum-ventral

75 ECM - elevated crossway maze

76 GSH - glutathione

77 GSH-Px - glutathione-peroxidase

$78 \mathrm{GSH}-\mathrm{Rd}$ - glutathione-redutase

79 GST - S-transferase glutathione

$80 \mathrm{HTAB}$ - hexadecyltrimethylammonium bromide

81 IGF-1 - insulin-like growth factor 1

$82 \mathrm{LT}$ - lateral tilting

83 LTM - long term recent memory duration

84 MD - mid-lateral

85 MLD - memory of long duration

86 MP - miloperoxidase enzymes

87 PGC-1 $\alpha$ - transcriptional coactivator peroxisome proliferator-activated receptor-

88 y coactivator $1 \alpha$

89 OD - optical density

90 ROS - reactive oxygen species

91 SD - superoxide-dismutase

92 STM - short term memory

$93 \mathrm{TMB}$ - tetramethylbenzene

94

95 INTRODUCTION 
96 Neuroinflammation is a complex process which consists in the answer of

97 immune cells, specialized in the central nervous system (CNS) to stimulation that

98 affects the basal activity of the parenchyma of such tissue [1]. However, the brain

99 is considered an immune privileged organ, since peripheral immune cells are

100 unable to cross the blood-brain barriers (BBB), limiting the neuroinflammatory

101 response to the CNS. Therefore, its interaction with the peripheral immune 102 system is little known [1, 2, 3].

103 Microglia cells are considered the immune cells of the CNS, and express 104 pro and anti-inflammatory effects, promoting the brain homeostasis [3]. In its 105 physiological basal activity, the microglia expresses an anti-inflammatory 106 phenotype and their ramifications go through continuous extension cycles, with 107 the purpose of monitoring the environment [2, 4].

108 However, when detecting a pathological stimulation, such as infections, 109 mechanical trauma, poorly folded proteins and ischemia [4], microglia quickly 110 modifies its morphology, expressing a reactive phenotype with pro-inflammatory 111 effects, synthesizing and secreting cytokines, such as TNF- $\alpha$ and IL-1 $\beta$ [5], and 112 activating nitric oxide synthesis -2 enzyme, cyclooxygenase 2 (COX2) and C-C 113 chemokine ligand 2 (CCL2), which produce reactive oxygen species (ROS) [6], 114 aiming at eliminating the aggressive stimulation and reparing the tissue damage $115[1,7]$.

It is known that the neuroinflammatory process is a needed mechanism 117 and important in the brain homeostasis [3]. However, when outside 118 (environmental) harmful persistent stimulation and/or internal stimulation are 119 recognized by the microglia [8], a system of positive retro-feeding is sustained, 
120

121

122

over-elevating the production of pro-inflammatory factors, which promote cell death and atrophy of local neural synapses, inducing symptoms such as cognitive decline, movement alterations and memory loss [4].

The constant activation of the microglia, the increase of the oxidative stress, the reduction of the neurotrophic support, the alteration of the metabolism of neurotransmitters and the rupture of the brain-blood barrier are described in the literature as associated mechanisms to pathologies and neuropsychiatric disorders, including Alzheimer's disease [6, 9], Parkinson disease and depression [5].

It is foreseen that neuroinflammatory/neurodegenerative diseases will be the biggest health concerns of this century and the second main cause of death by 2050 [10]. Being aware of such facts, several studies are performed with the purpose of developing therapeutic and preventive strategies that may mold the neuroinflammatory process. The practice of physical exercise, a nonpharmacological action, has shown to be promising in the search of reaching such goals [11], and has been proposed as an anti-oxidant and natural antiinflammatory strategy, which stimulates the increase of the hippocampal neuroplasticity [12, 3], hippocampal neurogenesis and cell proliferation [13], as well as it increases the synthesis of growth factors such as the brain derived neurotrophic factor (BDNF) [14, 15], insulin-like growth factor 1 (IGF-1) [16] and the production of enzymatic and non-enzymatic antioxidants [3].

All such benefits have a direct action in the symptomatology of patients with neuroinflammatory diseases, diminishing the cognitive decline, specially memory loss $[3,11]$. However, most relevant studies are focused on the benefits 
144 of aerobic exercises, and few studies approach the influence of muscular strength

145 training, specially when practiced prophylactically, with the purpose of preventing 146 a possible progression of a neuroinflammation, and how such effects are 147 modulated, and what the best strategy of physical training is, evaluating intensity, 148 training and recovery period.

149 This way, in the present study, we propose the investigation of the 150 neuroprotective effect of muscular strength training at a moderate intensity, 151 practiced prophylactically, in a neuroinflammation-induced model, induced by 152 lipopolysaccharide (LPS). For such, we analyzed the discriminative, averse and 153 social memory, and the activity of antioxidant enzymes in the hippocampus and 154 in the prefrontal cortex of mice which were submitted to the hippocampus intra155 CA1 infusion of LPS after 8 weeks of physical training.

156

\section{METHODS}

159 Animals and experimental design Male Wistar mice, of approximately 2 months old and with weight of 250 $350 \mathrm{~g}$ were used. The animals were conditioned in controlled room temperature at $22{ }^{\circ} \mathrm{C}$ with bright/dark cycles of 12 hours and kept in appropriate boxes with 163 capacity for 5 animals, covered with shaving, which was switched every two days. 164 They received water and food at ease. The maximum concern was deliberated 165 with the purpose of minimizing the suffering of the animals and reducing the 166 number of animals to be used in the research. All the experiments were according 
167

168

to the rules of the CEUA/UNIPAMPA and of the "Principles of laboratory animal care" (NIH publication $\mathrm{N}^{\circ} 85-23$, revised in 1996), under the report \#046/2017. For the experiments, the animals were divided into six groups, having ten animals per group. The groups were divided into control groups: group naive, group submitted to the intra-hippocampal infusion of saline, group submitted to the hippocampus infusion of LPS; and groups which practiced muscular strength exercises: group which performed only muscular strength exercises (CT), group which performed muscular strength exercises and was submitted to the infrahippocampal infusion of saline (exercise + saline), and group that performed muscular strength exercise and was submitted to the intra-hippocampal infusion of LPS (exercise + LPS).

The animals were submitted to eight weeks of training, three times a week.

After the final day of training, all animals performed the muscular strength testing by gripping through the Grip Strength equipment. After training, the surgery was performed to induce an initially acute neuroinflamation through LPS, which was infused in the CA1 region of the hippocampus. The post-operative period was of 5 days, followed by the beginning of the behavioral testings in the following sequence: open field, object recognition, social recognition, plus maze and hot plate. At last, the animals were euthanized, and then, the removal of the heart, the hippocampus and the prefrontal cortex was performed, to obtain the analysis of the activity of antioxidant enzymes.

\section{Strength exercise protocol}


Muscular strength training was performed using a personalized vertical

191 scale made of wood and iron $\left(1.1 \times 0.18 \mathrm{~m}, 2 \mathrm{~cm}\right.$ grip, inclination of $\left.80^{\circ}\right)$ with a 192 wooden box $(20 \times 20 \times 20 \mathrm{~cm})$ put in the upper part of the stairs. Initially, the 193 animals were familiarized with the exercise, performing four attempts of climbing 194 per day during three days. Once they were able to climb to the wooden box, they 195 could rest inside the wooden box for $120 \mathrm{~s}$. The strength training started a week 196 after the familiarization. In the first week, the load that corresponded to $50 \%$ of 197 the mouse's body mass was fixed at the base of the animal's tail. In the second 198 week of training, the test of maximum load was performed to determine the load 199 of exercise for each animal individually. To determine the maximum load 200 corresponding to $75 \%$ of the body mass and $30 \mathrm{~g}$ were added to each repetition 201 of addition climb until the mouse was not able to finish the exercise. The heavier 202 load transported successfully was considered the maximum load. The maximum 203 load was determined on the first day of each week of training. The training 204 sessions consisted of eight climbs in the stairs with 2 repetitions for each load of $20550 \%, 75 \%, 90 \%$ and $100 \%$ of the individual result of the maximum load, resulting 206 in 8 climbs with rest interval of 1 minute between the repetitions, with 3 training 207 sessions per week (one day for the maximum load and 2 days for the training 208 sessions), during 8 weeks [17].

\section{Grip Strength Meter}

After the final day of training, all animals performed the muscular strength

212 test by gripping through the equipment Grip Strength. The grip gauge was 213 positioned horizontally facing the equipment. The animals were put in the metal 
214 grid and, next, were pulled backwards in the horizontal plan by the tail. The forces

215 applied to the grid through the animal's legs were measured in grams and

216 immediately before losing the adherence the maximum tension was recorded.

217 Three tests were performed and as result, a mean value was obtained [18].

219 LPS administration

220 To induce the neuroinflammation process, the animals were submitted to

221 a stereotaxic surgery and subsequently the intra-hippocampal bilateral 222 administration (CA1 region) administration of vehicle (saline solution) or LPS 223 (from Escherichia coli 055:B5; Sigma) dissolved in PBS in the concentration of $22410 \mathrm{mg} / \mathrm{ml}$ and administrated in the dose of $40 \mathrm{~g} /$ side, through the infusion of 4 $225 \mathrm{l} /$ side during $10 \mathrm{~min}$. The coordinated used for the stereotaxic surgery was 226 adapted from the Paxinos \& Watson Anatomic Atlas, being as follows: antero227 posterior $(A P)=-4.2 \mathrm{~mm}$; mid-lateral $(M D)= \pm 3.0 \mathrm{~mm}$; dorsum-ventral $(D V)=-$ $2283.0 \mathrm{~mm}$; lateral tilting $(\mathrm{LT})=0^{\circ}$ starting from the Bregma point. The LPS infusion 229 was performed using a Hamilton $(5 \mu \mathrm{l})$ syringe. All the procedures were 230 performed with previously anesthetized animals with $75 \mathrm{mg} / \mathrm{kg}$ of ketamine and $23110 \mathrm{mg} / \mathrm{kg}$ of xylazine through the intra-peritoneal route. After the surgery, the mice 232 were put in housing boxes, under smooth heating, to avoid hypothermia.

234 Object recognition memory test

235 To evaluate the discriminative memory, the object recognition test was 236 performed. From the first to the fourth day of the experiment of such task, 237 considering the habituation period, the animal was put in the left superior corners 
238 of the device, a $50 \mathrm{~cm} \times 50 \mathrm{~cm} \times 50 \mathrm{~cm}$ box, made of compensated, acrylic 239 transparent polyvinyl chloride, and then left it there for 5 minutes, without a single 240 object inside the box, so that the animal could get used to the environment.

241 During the training session, one day after the last day of the habituation day,

242 the animal was once again put in the box with two equal objects, $A$ and $B$, located 243 right at the center of the box, and the animal was left there for 5 minutes, so it 244 could explore the environment freely. The exploration time of each object was 245 clocked for posterior evaluation. After 3 hours, the short term memory was tested, 246 in which the animal was replaced in the box, with two objects, object $A$ and object $247 \mathrm{C}$, relieving the previous object $\mathrm{B}$, in a period of time of 5 minutes for free 248 exploration. The long term memory duration was tested $24 \mathrm{~h}$ after the previous 249 test, in which the animal as once again placed inside the box, with two objects, 250 being one object $A$ and the other, object $D$, different from objects $A, B$ and $C$, and 251 the animal had 5 minutes for free exploration [20].

\section{Social recognition memory test}

The task of evaluating the social memory is an adaptation of the social 255 interaction test proposed by [21]. The task was performed in 3 days. First, the 256 animals were placed in a habituation field (the same size and characteristics 257 described in the previously described task of object recognition) with two small 258 cages during 20 minutes for free exploration. In the following day, the training 259 was performed with the inclusion of a juvenile mouse in one of cages for $1 \mathrm{~h}$ of 260 free exploration, being, at the same period, one cage left empty. After $24 \mathrm{~h}$, the

261 test was performed when the same mouse of the training (which means, the 
262 mouse that became familiar) and a new mouse were put for exploration for $5 \mathrm{~min}$.

263 The time spent exploring the new mouse and the familiar mouse was registered.

264 The exploration of the animal was defined as smelling or touching the cages with

265 the nose and/or front legs [19].

266

267 Open field

268 To verify whether the effects of the LPS or the vehicle infusion either

269 harmed or not the exploratory and moving activities, the animals were put for 5

270 minutes in the open field arena. The apparatus is from the same size and

271 characteristics previously described in the object recognition task. The

272 experiments were held in a low sound room under low intensity lighting. Each

273 mouse was placed in the center of the open field and the number of squares

274 crossed and rearing were registered [22].

275

276 Elevated plus maze

277 To evaluate the state of anxiety and to assure that the LPS or the vehicle

278 infusion did not harm such state, the mice were put in an elevated crossway maze

279 (ECM), and the number of entries and the time spent in the open and close ailes

280 were registered during a 5 minute session [23].

282 Hot plate

283 To evaluate the nociceptive response and the sensitivity of the animal's

284 legs and to assure that the LPS or the vehicle infusion would not harm the 285 nociceptive sensibility and, consequently, in the task of elusive inhibition, the hot 
286 plate test was use. The mouse was placed in a device with a warm metal sheet

287 of paper $\left(55 \pm 0,5^{\circ} \mathrm{C}\right)$ and the time until the animal showed reaction to the thermal

288 stimulation by raising or licking one's leg was determined [24].

289

290 Heart mass

291

After all the behavioral tasks, the animals were euthanized, and then the

292 collect of the hippocampus, prefrontal cortex and heart mass was performed. For

293 the heat mass collect, the open chest procedure was performed and blood was

294 drawn by cardiac puncture, followed by the removal of the heart. After such

295 removal, the heart was washed with saline solution $(0.9 \%)$ to remove the excess

296 of blood and, immediately the heart mass was weighed using an analytic scale.

297 The index of cardiac hypertrophy was calculated through the reason between

298 heart mass $(\mathrm{mg})$ and body mass $(\mathrm{g})$.

299

300 Preparation of the homogenate and protein analysis

301

Tissue samples of the hippocampus and from the prefrontal cortex were

302 weighed and homogenized with $200 \mathrm{mM}$ phosphate buffer. This homgenate was

303 used for the analysis of the quantification of the reduced glutathione (GSH)

304 concentration and of the miloperoxidase enzymes (MP), dismutase superoxide

305 (SD), catalase (CAT) and S-transferase glutathione (GST).

306 The protein concentration was measured with a spectrophotometer at

307 590nm using Bradford (Biorard®) reactant, and bovine albumin $(0.012-0.100$

$308 \mathrm{mg} / \mathrm{ml}$ ) was used for the standard curve. 
310

\section{Determination of MPO activity}

The homogenate obtained according to the previous description was centrifuged at $10000 \mathrm{rpm}$ for 20 minutes. The obtained precipitate was resuspended with $500 \mu \mathrm{L}$ of $80 \mathrm{mM}$ potassium phosphate buffer with $0.5 \%$ of hexadecyltrimethylammonium bromide (HTAB). After the homogenization, the samples were once again centrifuged at $12000 \mathrm{rpm}, 20$ minutes at $4^{\circ} \mathrm{C}$ of temperature in a high speed refrigerated microcentrifuge. In a 96-well plate in triplicate, aliquotes of $60 \mu \mathrm{l}$ of the supernatant of each sample, or distilled water for the white, and then $200 \mu$ l of a reactional solution $(100 \mu \mathrm{L}$ of $80 \mathrm{mM}$ phosphate buffer, $85 \mu \mathrm{l}$ of $22 \mathrm{mM}$ phosphate buffer and $15 \mu \mathrm{l}$ of $0.017 \% \mathrm{H}_{2} \mathrm{O}_{2}$ ) was added. The reaction stated with the addition of $20 \mu \mathrm{l}$ of tetramethylbenzene (TMB), an enzymatic substrate that results in a colorful product in each well. The plaque with the samples was incubated for $3 \mathrm{~min}$ at $37^{\circ} \mathrm{C}$, and the reaction was interrupted by the addition of $30 \mu \mathrm{l}$ of $1,46 \mathrm{M}$ sodium acetate $(\mathrm{pH}=3.0)$ in each well. The enzymatic activity was determined in a spectrophotometer at $620 \mathrm{~nm}$.

The results were expressed as units of optical density (OD)/mg of protein, according to [25].

\section{Quantification of the GSH and LOOH levels}

As described by [26, 27], $50 \mu \mathrm{l}$ of the homogenate was added to $40 \mu \mathrm{L}$ of $12,5 \%$ of thrichloroacetic. Next, the material was centrifuged at $1.4 \mathrm{G} / 15 \mathrm{~min}$. After the centrifuge, $20 \mu \mathrm{l}$ of the supernatant was added to $270 \mu \mathrm{l}$ of TRIS buffer (pH 8.9) and $10 \mu$ l of 5,5'-dithio-bis-[2-nitrobenzoic acid (DTNB). The absorbance 
333 was measured after $5 \mathrm{~min}$ in wavelength of $415 \mathrm{~nm}$ and the values were 334 interpolated in a standard deviation curve of GSH $(1.25-10.00 \mu \mathrm{g} / \mathrm{mL})$. The results 335 are expressed in $\mu \mathrm{g} / \mathrm{g}$ of tissue.

\section{Determination of SOD, CAT and GST activities}

The obtained homogenates were used to verify the participation of the

SOD, and it was based on the capacity of the SOD in inhibit the auto-oxidation of the pirogalol. In a conic tube, the following were added: $442,5 \mu$ l of Tris -EDTA buffer and $20 \mu \mathrm{l}$ of the sample. After agitation in a vortex, $25 \mu \mathrm{l}$ of $1 \mathrm{mM}$ pirogalol was added and incubated. Then, they were centrifuged and $300 \mu \mathrm{L}$ of the supernatant was pippeted in a microplate for the reading in the spectrophotometer at $205 \mathrm{~nm}$. The results were compared with the control group

345 (Tris-EDTA buffer with pirogalol without incubation + mean of the triplicate without

346 the sample and without incubation), being such value equal to $100 \%$. The amount

347 of protein that inhibits the reaction in $50 \%$ (IC 50) is equal to one unit (U) of SOD.

348 The results were expressed in $\mathrm{U}$ of SOD/mg of protein [28].

349 The homogenate was also used to verify the participation of the catalase 350 enzyme (CAT). In a 96-well microplate $10 \mu \mathrm{L}$ of the sample (homgenate) was 351 placed, added to 290 go of the reaction broth (5 mM Tris/EDTA buffer, $\mathrm{pH} 8.0+$ $35230 \% \mathrm{H}_{2} \mathrm{O}_{2}+$ ultra pure water) [29]. After being added all the elements, three 353 readings in the spectrophotometer were performed, at the wavelength of $240 \mathrm{~nm}$, 354 and the results were expressed in $\mu \mathrm{mol}$ of $\mathrm{H}_{2} \mathrm{O}_{2}$ consumed $/ \mathrm{min} / \mathrm{g}$ of tissue.

355 The supernatant was used to verify the presence of the S-transferase 356 glutathione enzyme. After centrifuge of the homogenate, the precipitate of the 
357 supernatant was separated. $50 \mu \mathrm{L}$ of the supernatant and $250 \mu \mathrm{L}$ of the reaction

358

359

360

361

362

363

364

365

366

367

368

369

370

371

372

373

374

375

376

377

378

379 broth (0,1M phosphate buffer, $3 \mathrm{mM}$ CDMB and $3 \mathrm{mM} \mathrm{GSH})$ were added in a 96well microplate. After such step, three reading in a spectrophotometer were performed at the wavelength of $340 \mathrm{mn}$, and the results were expressed in $\mu \mathrm{mol} / \mathrm{min} . / \mathrm{mg}$ of protein [30].

\section{Data analysis}

The latency and the time percentage of the behavioral experiments and the mean numbers obtained in the biochemical experiments were compared between the different groups through the parametric Student test (two groups) or ANOVA (more than two groups) with the post-hoc Newman-Keuls test for multiple comparisons, or Dunnett's test for the comparisons with the control group. P values $<0.05$ were considered statistically significant.

\section{RESULTS}

Data from the body weight were expressed in grams. All animals gained weight throughout the experiment. However, there was no significant difference regarding weight gain among the different groups: CT $(442.7 \pm 20.43)$, saline + exercise (419.8 \pm 16.47$)$, LPS + exercise (378.7 \pm 14.90$)$, Naive (445.4 \pm 14.88$)$ saline $(429.4 \pm 11.37)$ and LPS $(425.7 \pm 14.70)(p>0.05)$

With the purpose of evaluating the animal's maximum muscular strength and posteriorly prescribe the maximum load that the animal would use during the exercise, the animals submitted to the strength exercise protocol performed the 
380 maximum load test, once a week, during all the muscular strength training 381 protocol.

383 Figure 1. Eight weeks of strength exercise previously to the intra-CAl dorsal 384 bilateral infusion of LPS (40 $\mu \mathrm{g} / \mathrm{side})$ or saline improved the muscular strength of 385 the animals.

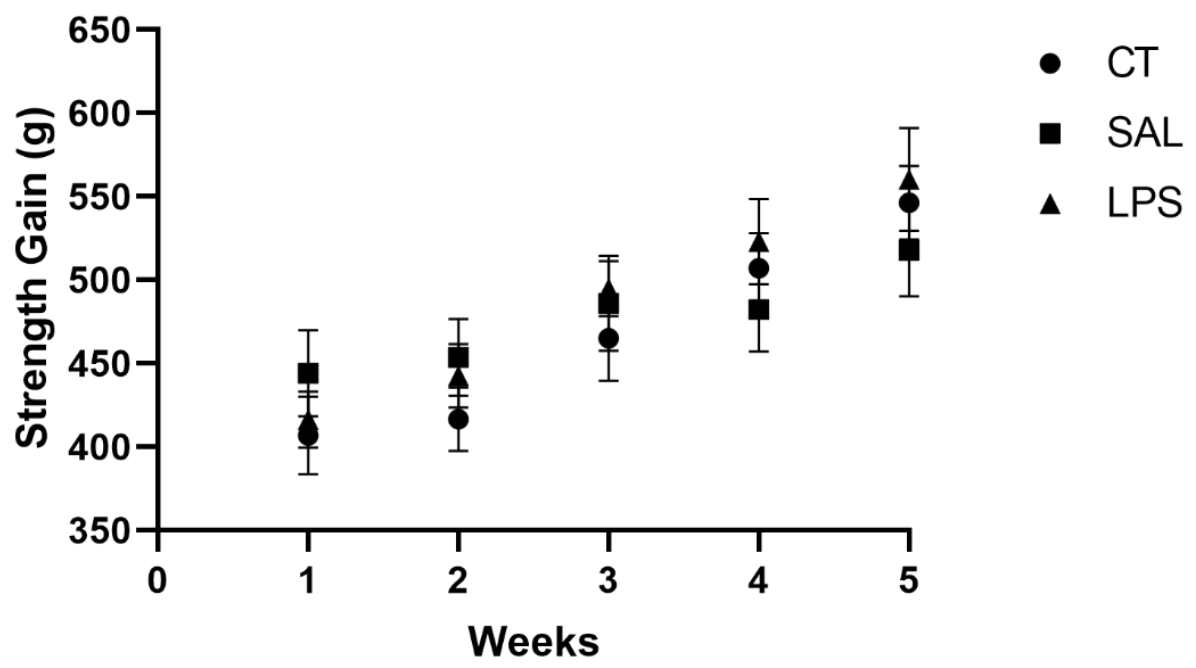

The animals were submitted to the maximum load test once a week during 389 all the muscular strength training protocol. The animals achieved gradual 390 muscular gain. However, there was not statistical differences among the groups 391 ( $p>0.05$, two-way ANOVA, followed by the Newman-Keuls multiple comparison 392 test).

As seen in figure 1, the prophylactic intervention with exercise resulted in 394 progressive muscular strength increase in the animals that trained for 8 weeks. 395 However, it was not verified significant difference among the trained groups that 396 received LPS/saline or the control group (CT). 


\section{GRIP STRENGTH TESTING}

After the final day of muscular strength training, the animals were

400 submitted to the grip strength testing through the equipment Grip Strength Meter.

401 The results of the outcome of the test for all the groups are shown in Figure 2.

402

403 Figure 2. Eight weeks of strength exercise previously to the infusion of intra-CA1 404 dorsal bilateral infusion of LPS $(40 \mu \mathrm{g} / \mathrm{side})$ induced an increase in the grip 405 strength.

406

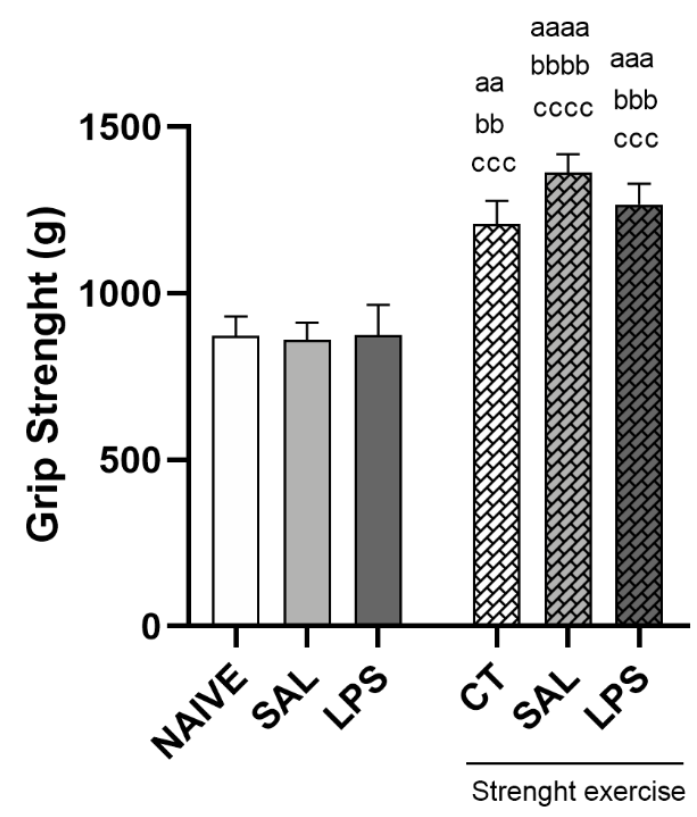

407

408

The mice were submitted to eight weeks of strength exercise training 409 protocol. As followed, they received intra-CA1 dorsal bilateral infusion of either 410 saline (SAL) or LPS (40 $\mathrm{gg} / \mathrm{side})$. Groups NAIVE and control (CT) were not 411 submitted to the procedure of intra-CA1 infusion. One day after the final day of 
412 training, the animals were submitted to the grip strength test $(\mathrm{g})$, through the 413 equipment Grip Strength Meter. Analysis of variance two-way ANOVA, followed 414 by Newman-Keuls multiple comparison test. aa $P<0.01$; aaa $P<0.001$; aaaa $P$ $415<0.0001$, when compared with the group NAIVE. bb $P<0.01$; bbb $P<0.001$; 416 bbbb $P<0.0001$ when compared with the SAL group, when not submitted to 417 exercise. ccc $P<0.001$; $\operatorname{cccc} P<0.0001$ when compared to the LPS group, when 418 not submitted to exercise. As expected, the prophylactic intervention with exercised resulted in grip

420 strength increase in the trained animals, demonstrating that the proposed training 421 protocol in the present study was efficient, significantly increasing the muscular 422 strength of the animals that were trained, when compared to the animals which 423 were not trained.

\section{OPEN FIELD}

426 The animals had their locomotor and exploratory capacity analyzed in the 427 open field task, during the habituation phase which preceded that object 428 recognition task. The results of the performance in such task for all the groups 429 are shown in Figure 3.

430

431 Figure 3. Eight weeks of strength exercise previous to the intra-CA1 dorsal 432 bilateral infusion of LPS $(40 \mu \mathrm{g} / \mathrm{side})$ does not affect the locomotion and 433 exploratory activity of mice in the open field task. 


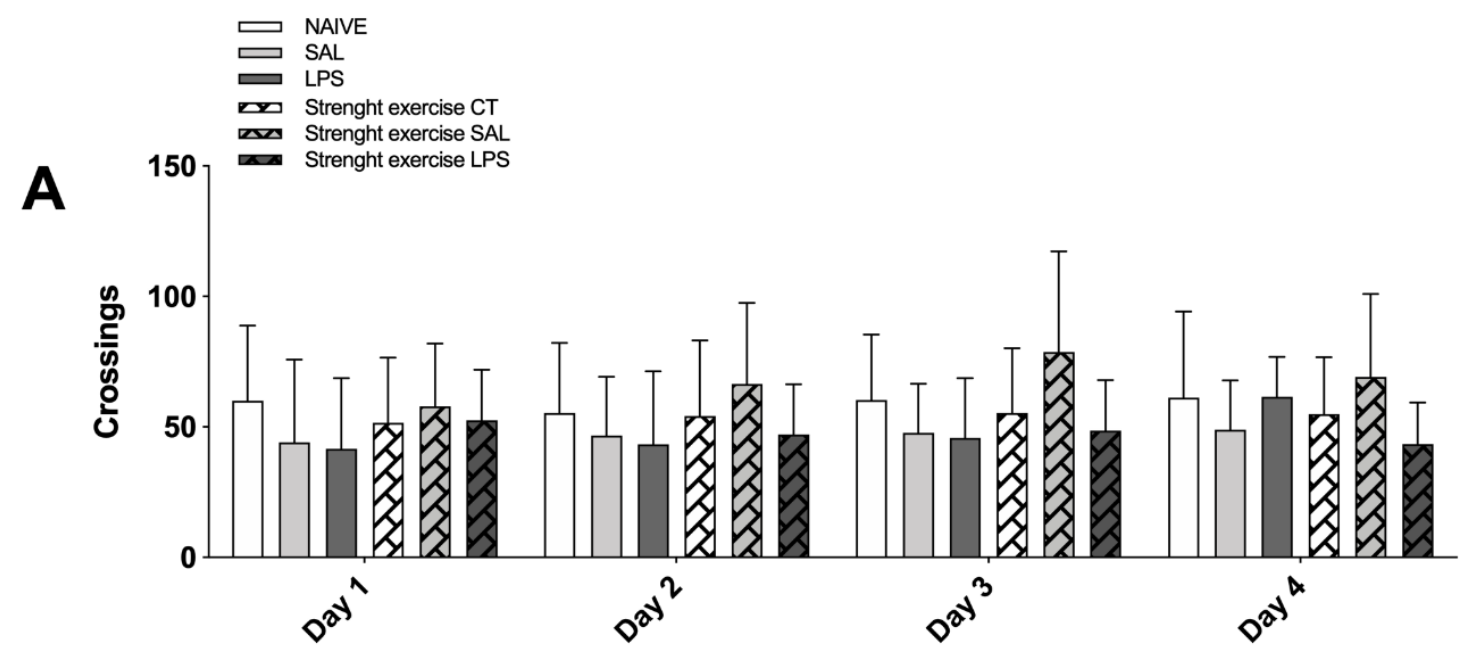

B
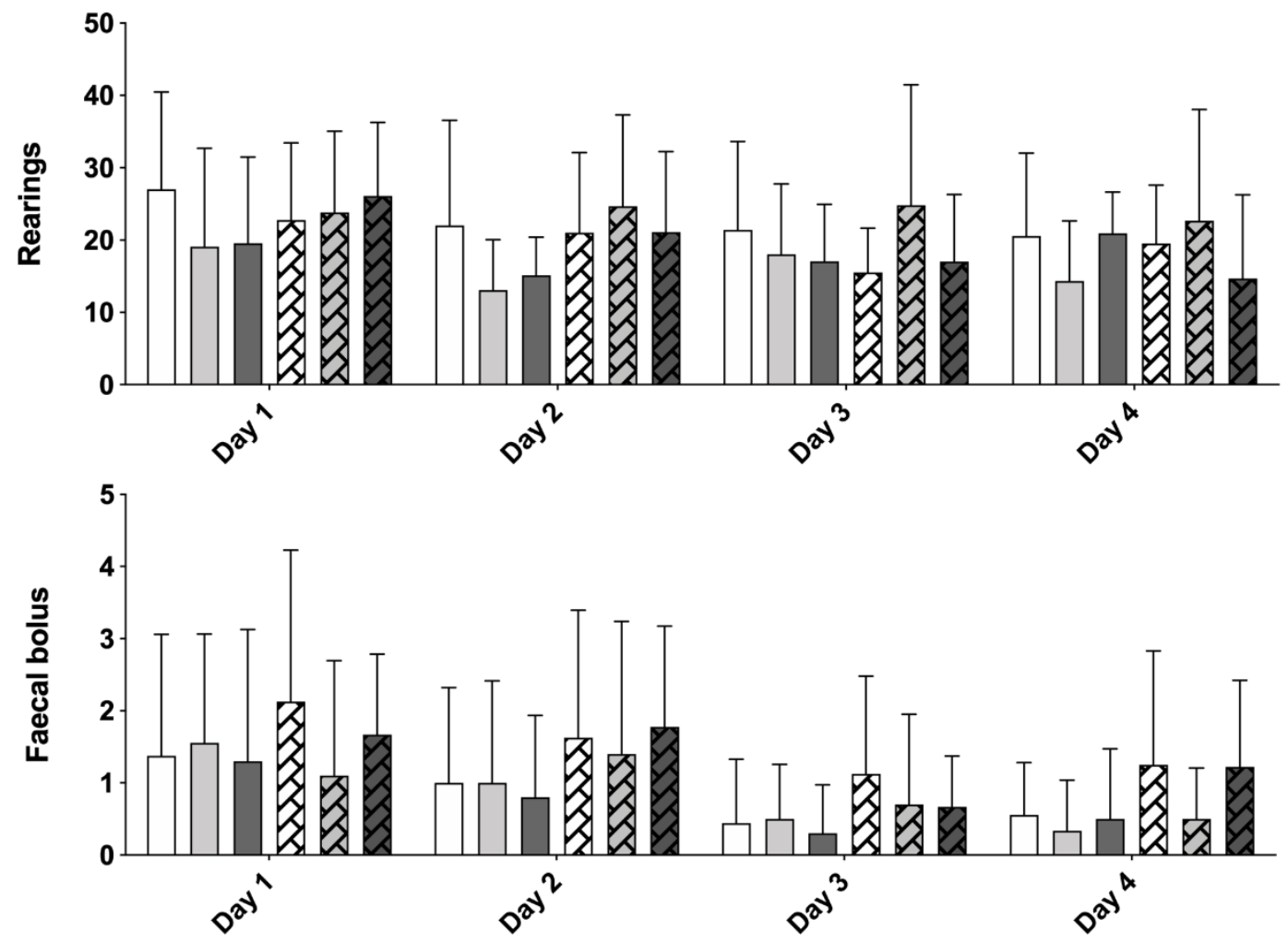

437 protocol. As followed, they received intra-CA1 dorsal bilateral infusion of either 438 saline (SAL) or LPS (40 $\mu \mathrm{g} /$ side). The groups NAIVE and control (CT) were not 439 submitted to the procedure of intra-CA1 infusion. After 5 days of postoperative 
440 recovery, these mice were submitted to several behavioral tasks, among them

441 the open field task, as part of the habituation process which preceded the object

442 recognition task. Four sessions were performed, one by day, during 4

443 consecutive days. (A) Number of crossings among internal squares of the open

444 field box (B) Number of times that the mouse lifted its back legs. (C) Number of

445 feces expelled by the mouse during the period of session in open field. Data are

446 expressed as mean numbers ( \pm EM) from the previous variables. There was not

447 significant statistical difference among the groups, after two-way ANOVA with

448 repeated measurements, followed by the Newman-Keuls multiple comparison 449 test.

450 There were not significant differences among the groups throughout the 451 days in the analyzed parameters analyzed in the open field task. Therefore, 452 neither the resistance exercise nor the neuroinflammation induced by the LPS in 453 the CA1 hippocampus region were able to alter the locomotor and exploratory 454 activity of mice in the open field task regarding the control group. 455

456 OBJECT RECOGNITION

457 The animals had their short and long term retention duration of the 458 discriminative memory analyzed in the object recognition task. The results of the 459 performance in such task are shown in Figure 4. 460

461 Figure 4. Eight weeks of strength exercise previous to the intra-CA1 dorsal 462 bilateral infusion of LPS (40 $\mu \mathrm{g} / \mathrm{side})$ improves the performance of mice in the 
463 retention of the short term memory (STM) or long term recent memory duration 464 (LTM) of discriminative memory relative to the object recognition task.
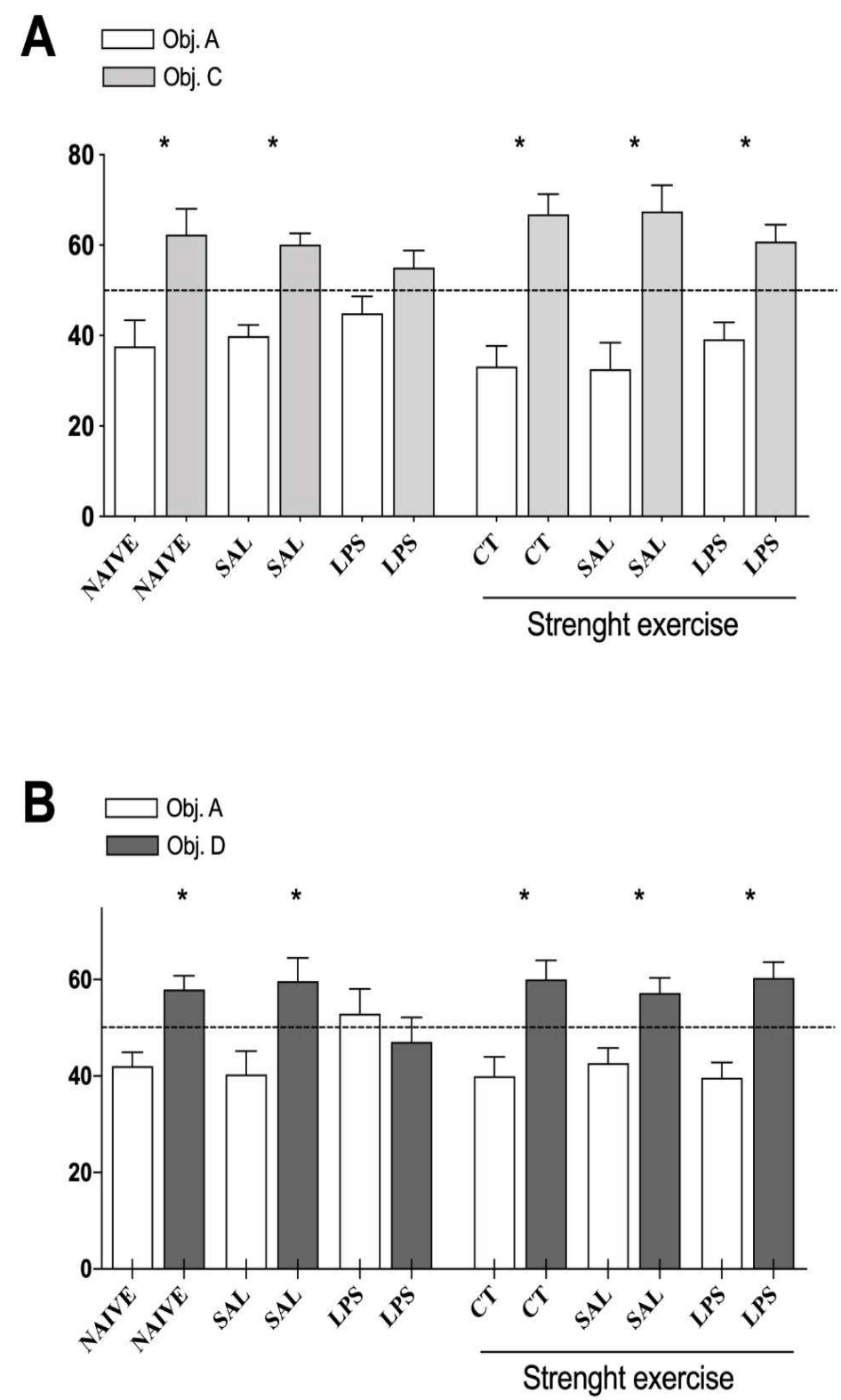

465

The mice were submitted to eight weeks of training in the strength exercise

468 training protocol. As followed, they received intra-CA1 dorsal bilateral infusion of 469 either saline (SAL) or LPS (40 $\mu \mathrm{g} /$ side). After 5 days of postoperative recovery, 470 these mice were submitted to several behavioral tasks, among them the object 
471 recognition task. (A) Test performed 3 hours after the training session with the

472 objects A and B. (B) Second test, performed 24 hours after the training session.

473 Data are expressed as mean numbers ( $\pm E M)$ from the exploration percentage of

474 each of the objects presented regarding the total time of the exploration of the 475 objects. ${ }^{*} p<0.05$ vs. Theoretical percentage of $50 \%$ in Student's $t$ test. ( $n=7-$ 47610 per group).

477 As expected, the locally provoked neuroinflammation in the CA1 478 hippocampus region harmed the mice capacity to form and retain descriminitative 479 short and long term duration regarding object recognition task. The prophylactic 480 intervention with strength exercise resulted in a protection effect, which was 481 verifiable both in the retention of the discriminating short term memory and recent 482 long duration. Therefore, in this cognitive task, as well as what happened in the 483 social recognition task, the strength exercise has shown to have potential 484 prophylactic mnemonic neuroptrotector in face of a local neuroinflammatory 485 effect.

486

487 SOCIAL RECOGNITION

488 The animals had their retention capacity of short and long duration of 489 social memory analyzed in the social recognition task. The results of the 490 performance in such task for all the groups are shown in Figure 5. 491

492 Figure 5. Eight weeks of strength exercise, previously to the intra-CA1 dorsal 493 bilateral infusion of LPS $(40 \mu \mathrm{g} / \mathrm{side})$, improves the performance of mice in the 
494 retention of social recent memory of long duration (MLD) regarding the task of 495 social recognition.

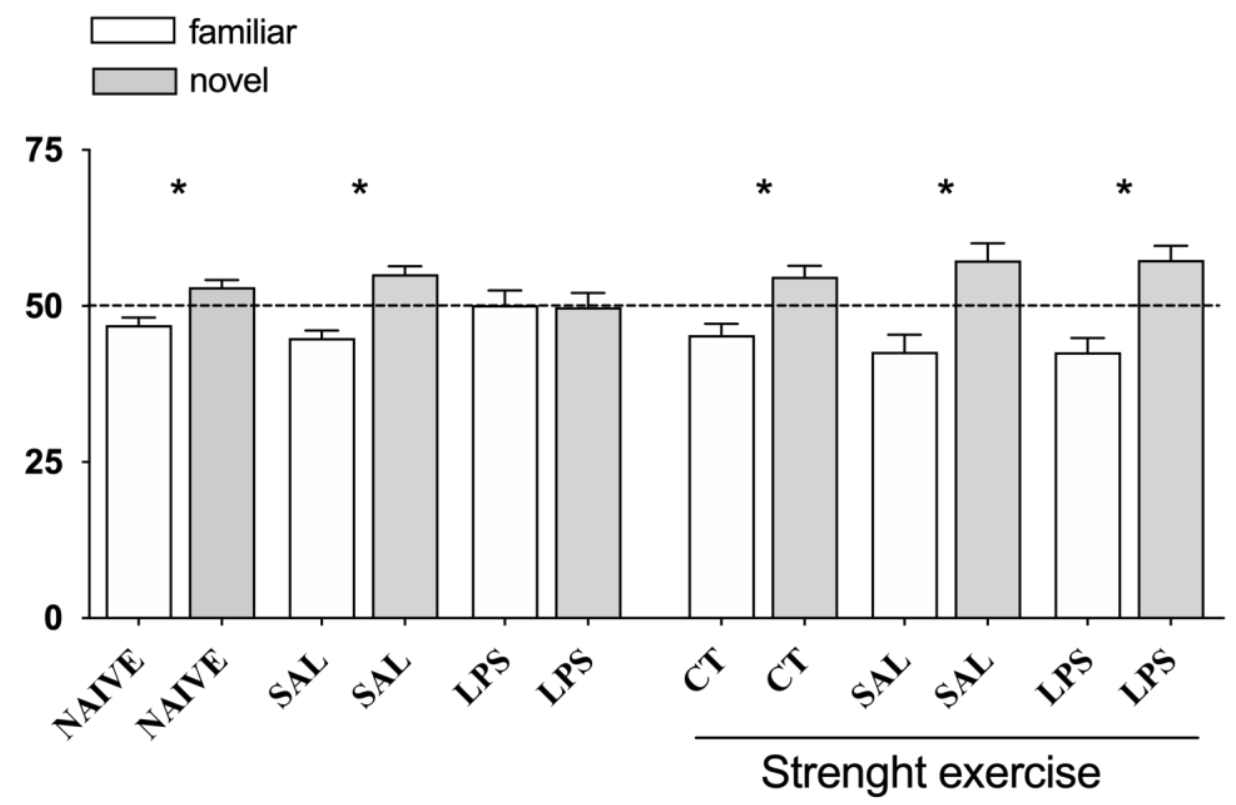

496

497

498

The mice were submitted to eight weeks of training in the strength exercise

499 protocol. As followed, they received intra-CA1 dorsal infusion of either saline 500 (SAL) or LPS $(40 \mu \mathrm{g} / \mathrm{side})$. The groups NAIVE and control (CT) were not 501 submitted to the intra-CAl infusion procedure. After 5 days of post-operative 502 recovery, these mice were submitted to several behavioral tasks, among them 503 the social recognition task. Test performed 24 hours after the training session. 504 Data are expressed as mean numbers ( $\pm E M)$ from the percentage of the 505 exploration of each of one of the juvenile mice regarding the total time of 506 exploration of such mice. ${ }^{*} p<0.05$ vs. Theoretical percentage in $50 \%$ in t Student 507 test. ( $n=7-10$ per group). 
As expected, the locally provoked neuroinflammation in the CA1

510 hippocampus region harmed the capacity of the mice to form and retain social

511 memory of short and long duration related to the social recognition task. The

512 prophylactic intervention with strength exercise resulted in a verifiable protecting

513 effect in the retention of the discriminating memory of recent long duration.

514 Therefore, in this cognitive tasks, the strength exercise has shown to be have

515 prophylactic neuroprotective potential in face of an acute neuroinflammatory

516 context.

517

518 PLUS MAZE

519 The animals had their level of anxiety analyzed in the elevated plus maze.

520 The results of the performance in such task for all groups are shown in Figure 6.

521

522 Figure 6. Eight weeks of strength exercise previously to the intra-CA1 dorsal 523 bilateral infusion of LPS ( $40 \mu \mathrm{g} / \mathrm{side})$ does not affect the level of anxiety of mice 524 in the plus maze elevated task. 
A

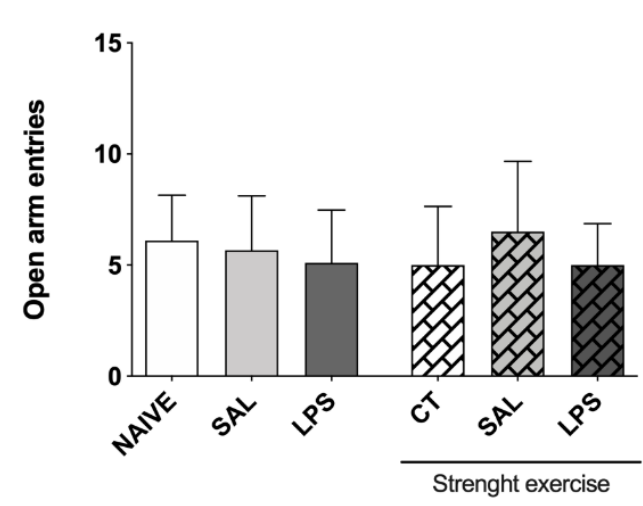

C

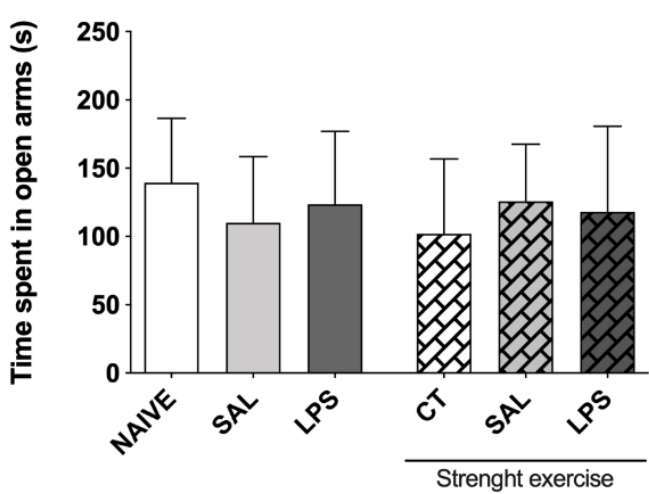

B

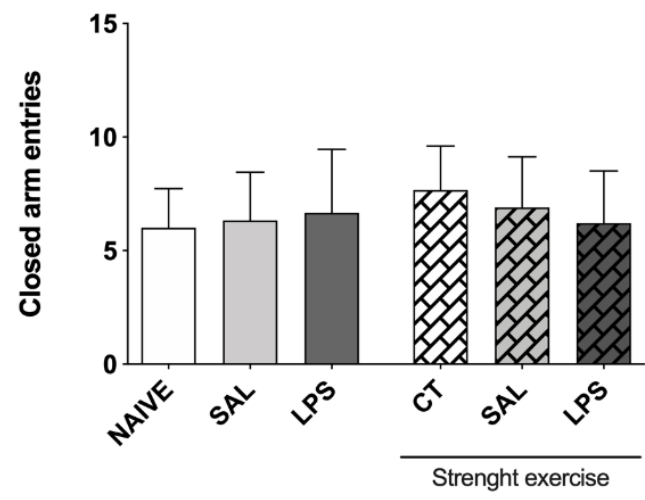

D

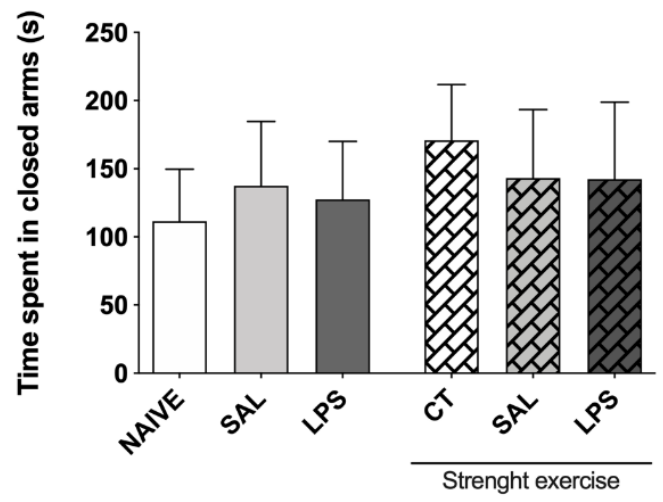

The mice were submitted to two weeks of training protocol in the strength

exercise. As followed, they received intra-CA1 dorsal bilateral infusion of saline

529 (SAL) or LPS $(40 \mu \mathrm{g} / \mathrm{side})$. The groups NAIVE and control (CT) were not

530 submitted to the intra-CA1 infusion procedure. After 5 days of postoperative

531 recovery, these mice were submitted to several behavioral tasks, among them

532 the elevated plus maze task. (A) Number of entries in the open arms. (B) Number

533 of entries in the closed arms. (C) Time spent within the open arms. (D) Time spent

534 within the closed arms. Data are expressed as mean numbers ( \pm EM) from

535 previous variables. There were no significant difference among the groups,

536 whether after one-way ANOVA followed by multiple comparison Student- 
537 Newman-Keuls test, whether after comparisons with the NAIVE group, according 538 to the test of Dunnett.

540 parameters in the elevated plus maze task. Therefore, neither the strength

541 exercise nor the neuroinflammation induced by the LPS in the CA1 hippocampus

542 region were able to alter the level of anxiety in the mice in such task regarding to 543 the control groups.

\section{HOT PLATE}

546 The animals had their nociception analyzed in the hot plate task. The 547 results of the performance in such task for all the groups are shown in Figure 7. 548

549 Figure 7. Eight weeks of strength exercise previously to the itra-CA1 dorsal 550 bilateral infusion of LPS $(40 \mu \mathrm{g} / \mathrm{side})$ does not affect the nociception of mice in 551 the hot plate task.

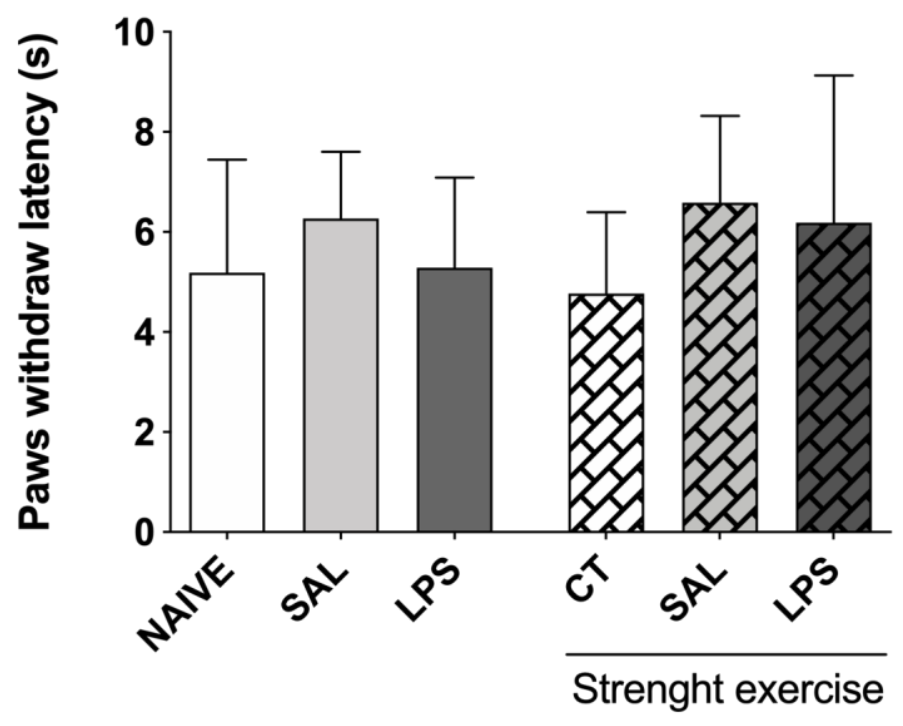


The mice were submitted to eight weeks of training in the strength exercise

554 protocol. As followed, they received the intra-CA1 dorsal bilateral infusion of

555

556

557

558

559

560

561

562

563

564

565

566 567 mice in this task regarding the control groups.

568

569

570

571

572

573

574

575 exercise.

\section{HEART MASS} saline (SAL) or LPS (40 $\mu \mathrm{g} / \mathrm{side})$. The groups NAIVE and control (CT) were not submitted to the intra-CA1 infusion procedure. After 5 days of post-operative recovery, these mice were submitted to several behavioral activities, among them the hot plate task. The data are expressed as mean numbers ( $\pm E M)$ of the reaction time to the thermal stimulation (removal and lick of the legs). There was not statistical significant difference among the groups, whether after one-way ANOVA followed by the Student-Newman-Keuls multiple comparison test, or after the comparisons with the NAIVE group, according to the Dunnett's test. There was not significant statistical difference among the groups regarding the reaction time to the thermal stimulation, by removing and licking the legs. Therefore, neither the strength exercise nor the induced neuroinflammation by the LPS in the CA1 hippocampus region were able to alter the nociception of the

Figure 8 presents the data referring to the heart mass, presented as reason between mass of the heart by body mass of the animal, index which determines the occurrence of cardiac hypertrophy. The reason heart mass/body mass from the group CT, SAL + exercise and LPS + exercise was significantly higher than the respective groups NAIVE, SAL without exercise and LPS without 
577 Figure 8. Eight weeks of strength exercise previously to the intra-CA1 dorsal

578

579

580

581

582

583

584

585

586

587

588

589

590

591

592

593

bilateral infusion of $(40 \mu \mathrm{g} / \mathrm{side})$ induces a physiological cardiac hypertrophy caused by muscular training.

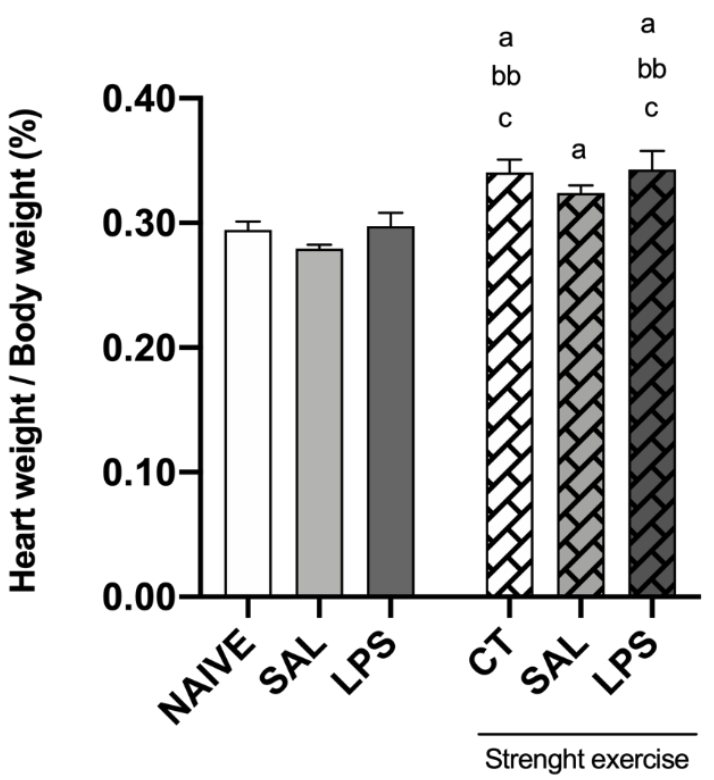

The mice were submitted to eight weeks of training in the strength exercise protocol. As followed, they received the intra-CA1 dorsal bilateral infusion of saline (SAL) or LPS (40 $\mu \mathrm{g} / \mathrm{side})$. The groups NAIVE and control (CT) were not submitted to the intra-CA1 infusion procedure. After being euthanized, the heart of the animals was removed and then weighted. The heart mass was corrected by the body mass. For such correction, the following formula was used: (Total weight of the heart/body weight) $x$ 100. Two-way ANOVA, followed by NewmanKeuls multiple comparison test, a $P<0.05$ when compared to the group NAIVE. bb $P<0.01$ when compared to the group SAL not exercised. $c P<0.05$ when compared to the group LPS not exercised.

The prophylactic intervention of muscular strength exercise was able to induce a physiological hypertrophy, compensatory/adaptation, needed to 
594 maintain a cardiac performance in increased condition of circulatory overload 595 during the animals' training. It is known that the intensive and long-lasting 596 physical training induces cardiovascular adaptations, including a cardiac 597 hypertrophy, which allows the heart an exceptional physical performance. We 598 noticed, in this study, a statistical difference in the heart mass of the animals 599 which trained, when compared to the ones that did not train, which shows that 600 the heart of the trained animals became adapted to the muscular strength 601 training, showing that the proposed training protocol in the present study was 602 efficient.

603

604 Evaluation of the effect of strength exercise on GSH level in rat 605 hippocampus after intra-hippocampal infusion of LPS.

606

With the purpose of knowing whether the maintenance of the intracellular 607 level of GSH is influenced by the practice of prophylactic strength exercise, after 608 a local neuroinflammatory condition, the hippocampus concentration of GSH was 609 evaluated in hippocampus samples of mice. Figure 9 shows that the 610 hippocampus concentration of GSH in animals which were submitted to the 611 strength exercise was smaller than in the group that received LPS intra612 hippocampus infusion, when compared to the group that received only LPS intra613 hippocampus infusion $(p<0.05)$ but was not previously submitted to the strength 614 exercise.

615 Figure 9. Evaluation of the influence of the strength exercise in the hippocampal 616 concentration of GSH. Eight weeks of strength exercise previously to the intra617 CA1 dorsal bilateral infusion of LPS (40 $\mu \mathrm{g} /$ side) diminished the hippocampus 
618 concentration of GSH in the mice hippocampus previously submitted to the 619 strength exercise, when compared to the non-exercised mice.

620

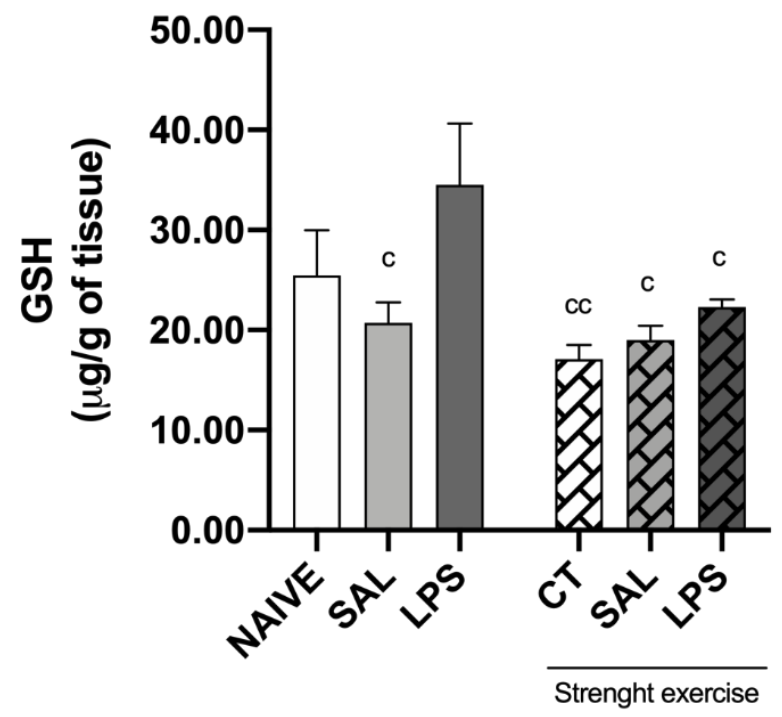

622

The mice were submitted to eight weeks of training in the strength exercise protocol. As followed, they received intra-CA1 dorsal bilateral infusion of either saline (SAL) or LPS (40 $\mu \mathrm{g} /$ side). The groups NAIVE and CT were not submitted

626 to the intra-CA1 infusion procedure. After 5 days of post-operative recovery, such

627 mice were submitted to several behavioral tasks, followed by euthanasia and then

628 the removal of the hippocampus was performed. Results expressed in mean

629 numbers \pm EPM. ( $\mathrm{n}=7-10$ per group). c $\mathrm{P}<0.05$, cc $\mathrm{P}<0.01$ when compared 630 to the non-exercised LPS group (One way ANOVA followed by Newman-Keuls 631 multiple comparison test). 
636 activity in the hippocampus in animals submitted to the intra-hippocampal 637 infusion of LPS when compared to the respective control group (LPS without 638 exercise).

639

640 Figure 10. Influence of the strength exercise in the activity of MPO in the 641 hippocampus. Eight weeks of strength exercise previously to the intra-CA1 dorsal 642 bilateral of LPS (40 $\mu \mathrm{g} / \mathrm{side})$ does not affect the activity of MPO in the 643 hippocampus.

644

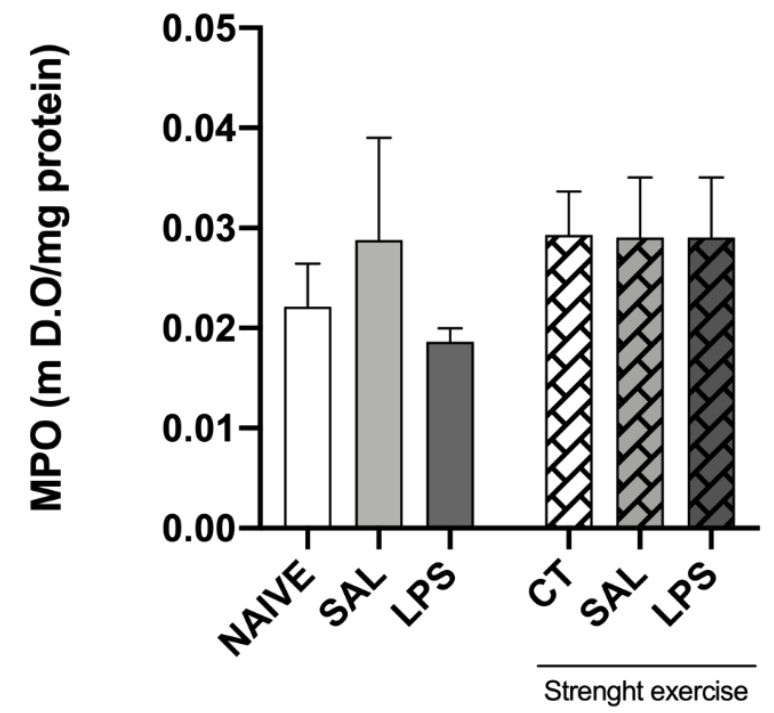

646

647 The mice were submitted to eight weeks of training in the strength exercise 648 protocol. As followed, they received intra-CA1 dorsal bilateral infusion of either 649 saline (SAL) or LPS (40 $\mu \mathrm{g} / \mathrm{side})$. The groups NAIVE and CT were not submitted 650 to the intra-CA1 infusion procedure. After 5 days of post-operative recovery, such 651 mice were submitted to several behavioral tasks, followed by euthanasia and then 
652 the removal of the hippocampus was performed. Results expressed in mean 653 numbers \pm EPM. ( $n=7-10$ per group) and statistical analysis was performed by 654 one-way ANOVA, followed by Newman-Keuls multiple comparison test.

655

656 Effect of strength exercise on the expression of the SOD enzyme in the 657 hippocampus of rats after intra-hippocampal infusion of LPS

658 As shown in Figure 11, the strength exercise was not able to re-establish 660 the activity of the enzyme SOD in mice submitted to the intra-hippocampal 661 infusion of LPS when compared to the control group.

662

663 Figure 11. Influence of the strength exercise in the activity of the enzyme SOD.

664 Eight weeks of strength exercise previously to the intra-CA1 dorsal bilateral 665 infusion of LPS (40 $\mu \mathrm{g} / \mathrm{side})$ does not affect the activity of SOD in the 666 hippocampus of mice.

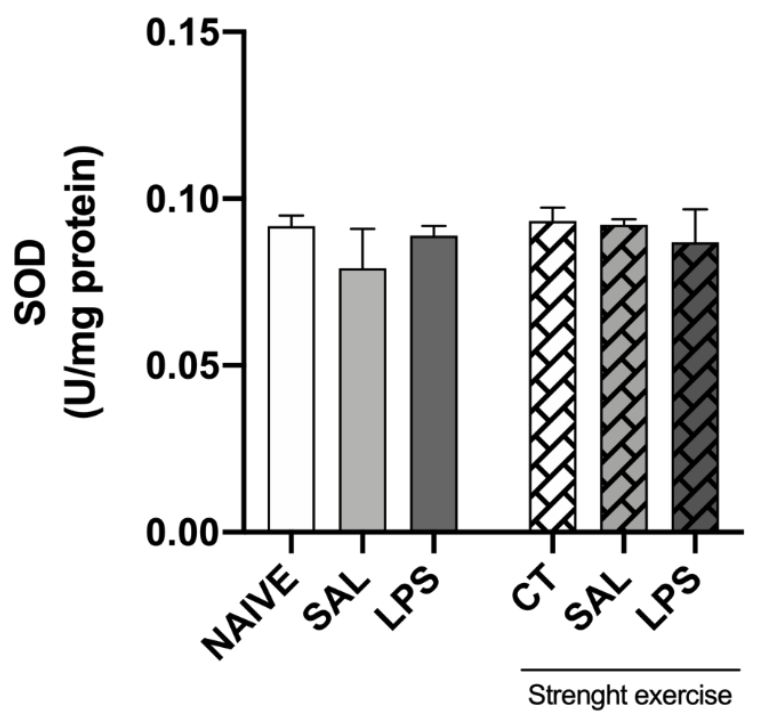


669

670

671

672

673

674

675

676

677

678

679

680

681

682

683

684

685

686

687

688

689

690 infusion of LPS ( $40 \mu \mathrm{g} / \mathrm{side}$ ) increased the activity of CAT in the hippocampus of

691 mice which received local induction of neuroinflammation. 


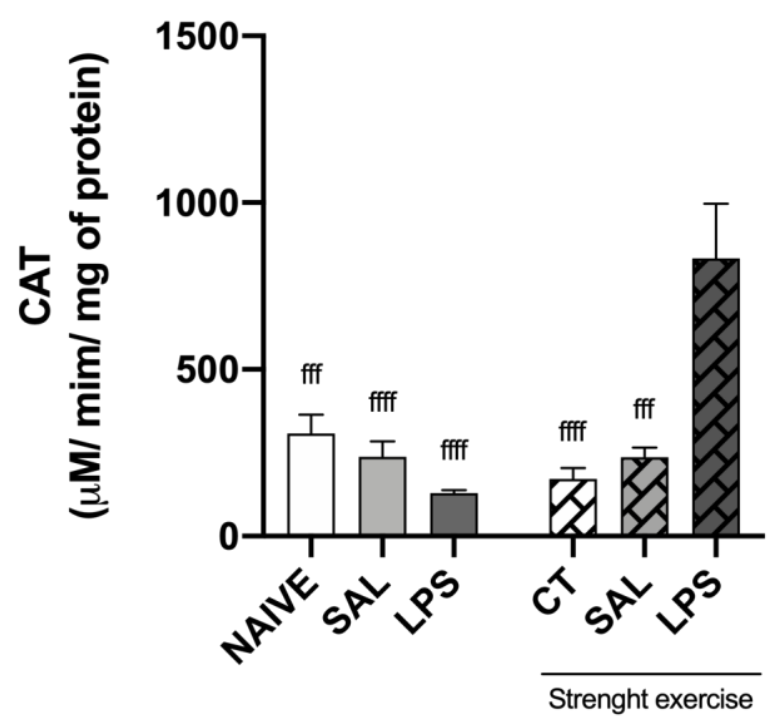

693

The mice were submitted to eight weeks of training in the strength exercise protocol. As followed, they received intra-CA1 dorsal bilateral infusion of either 696 saline (SAL) or LPS (40 $\mu \mathrm{g} / \mathrm{side})$. The groups NAIVE and CT were not submitted 697 to the intra-CA1 infusion procedure. After 5 days of post-operative recovery, such 698 mice were submitted to several behavioral tasks, followed by euthanasia and then 699 the removal of the hippocampus was performed. Results expressed in mean 700 numbers \pm EPM. ( $n=7-10$ per group) fff $P<0.001$, ffff $P<0.0001$ when compared 701 to the exercised LPS group, in one-way ANOVA followed by Newman-Keuls 702 multiple comparison test.

704 Effect of strength exercise on the expression of the GST enzyme in 705 hippocampus in rats after intra-hippocampal infusion of LPS

Figure 13 shows that the strength exercised influenced the activity of the 707 enzyme GST. We observed that the group which performed strength exercise 708 and was submitted to the intra-hippocampal infusion of LPS showed higher 
709 activity of the enzyme GST when compared to the group which practiced strength

710 exercise and received intra-hippocampal infusion of saline and the group that

711 only practiced physical strength. $(\mathrm{P}<0.05)$.

712

713 Figure 13. Influence of the strength exercise in the activity of the enzyme GST.

714 Eight weeks of strength exercise previously to the intra-CA1 dorsal bilateral 715 infusion of LPS (40 $\mu \mathrm{g} / \mathrm{side}$ ) does not affect the activity of GST in the 716 hippocampus of mice.

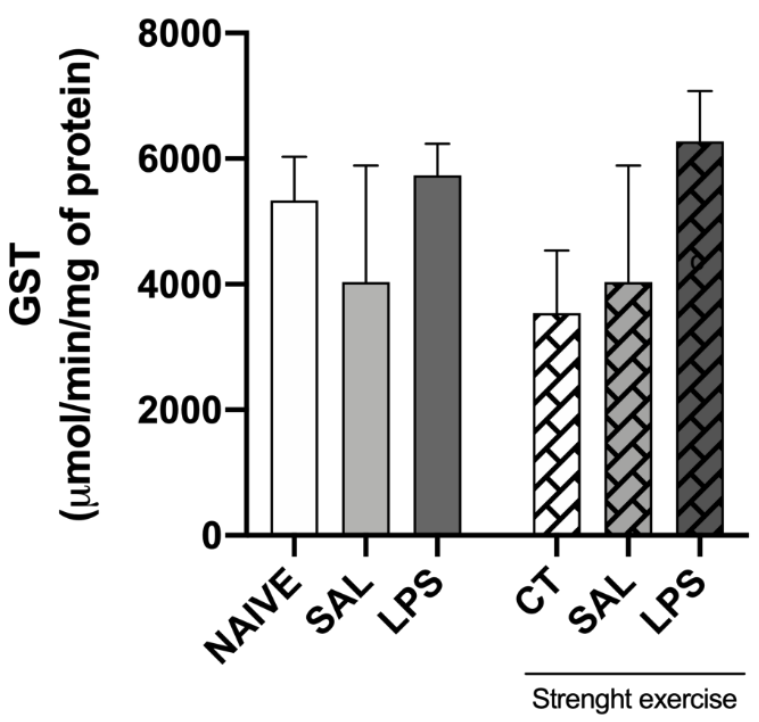

The mice were submitted to eight weeks of training in the strength exercise

720 protocol. As followed, they received intra-CA1 dorsal bilateral infusion of either

721 saline (SAL) or LPS (40 $\mu \mathrm{g} / \mathrm{side})$. The groups NAIVE and CT were not submitted

722 to the intra-CA1 infusion procedure. After 5 days of post-operative recovery, such

723 mice were submitted to several behavioral tasks, followed by euthanasia and then

724 the removal of the hippocampus was performed. Results expressed in mean 
725 numbers \pm EPM. ( $n=7-10$ per group) and statistical analysis by one-way ANOVA 726 and Newman-Keuls multiple comparison test.

728 Effect of strength exercise on the expression of MPO E GSH and CAT, SOD 729 and GST enzymes in the prefrontal cortex of rats after infusion of LPS in 730 the CA1 region of the hippocampus which practiced strength exercise without the induction of intra-CA1 hippocampal 734 neuroinflammation, when compared to the group NAIVE $(p<0.05)$ and the group 735 LPS exercised $(p<0.05)$.

736

737 Figure 14. Expression of GSH and from the enzymes SOD, CAT, GST and MPO 738 in prefrontal cortex of mice. Eight weeks of strength exercise diminished the 739 activity of CAT in the prefrontal cortex of mice, in relation to non-exercised mice, 740 except when the mice were submitted to intra-CA1 dorsal bilateral infusion of LPS $741(40 \mu \mathrm{g} / \mathrm{side})$ soon after the period of exercise. 


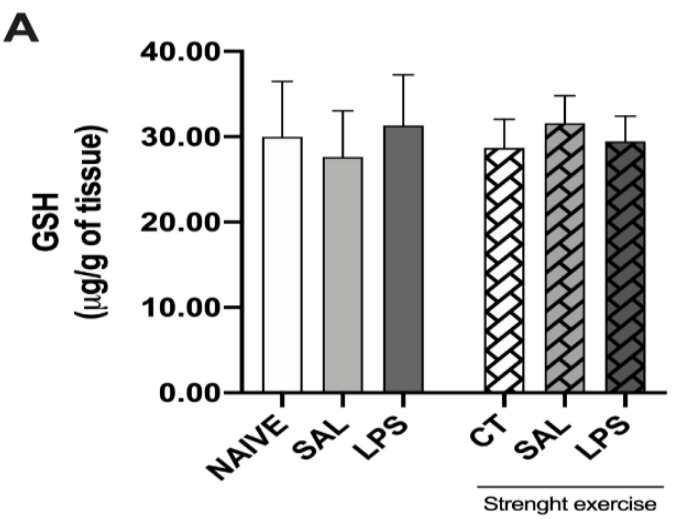

B

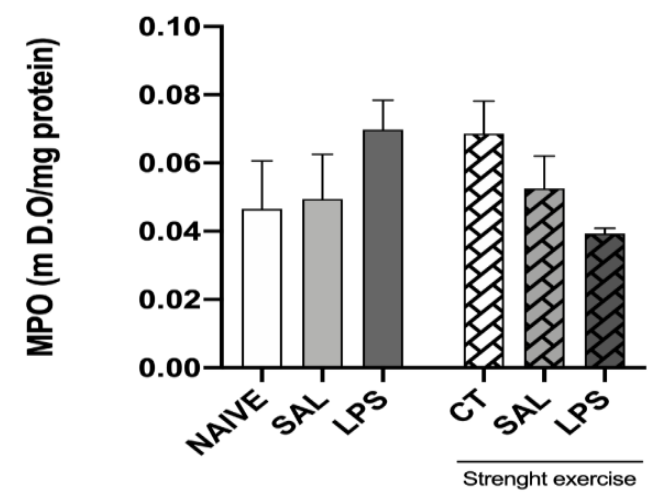

D

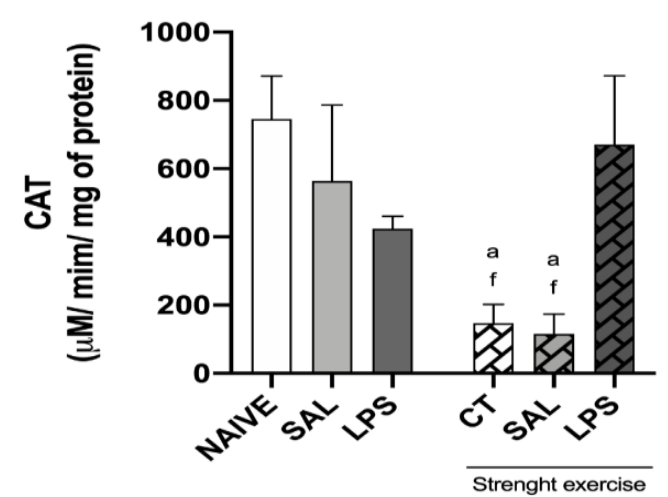

C

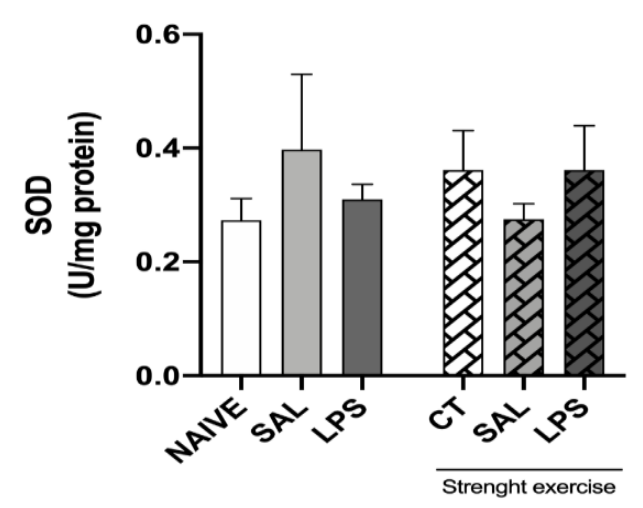

E

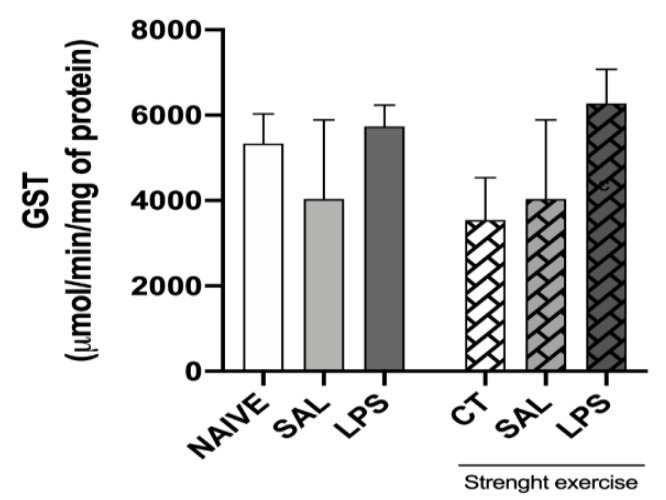

The mice were submitted to eight weeks of strength exercise training

745 protocol. As followed, they received intra-CA1 dorsal bilateral infusion of either

746 saline (SAL) or LPS (40 $\mu \mathrm{g} / \mathrm{side})$. The groups NAIVE and CT were not submitted

747 to the intra-CA1 infusion procedure. After 5 days of post-operative recovery, such

748 mice were submitted to several behavioral tasks, followed by euthanasia and then 
749 the removal of the prefrontal cortex was performed. Panel (A-E) showing the 750 concentration of GSH GSH (A) and the activity of the enzymes MPO (B), SOD 751 (C), CAT (D) and GST (E). Results expressed as mean numbers \pm EPM. ( $n=7$ 75210 per group). Figure D: a $\mathrm{P}<0.05$ when compared to the group NAIVE; $\mathrm{f}<<$ 7530.05 , when compared to the group LPS which practiced strength exercise. One754 way ANOVA followed by Newman-Keuls multiple comparison test.

\section{DISCUSSION}

With the purpose of investigation the influence of muscular strength exercise practiced prophylactically on the parameters of oxidative stress and memory deficits caused by local neuroinflammation in a key brain structure to the formation and consolidation of memories [31], we showed that the muscular strength exercise has neuroprotective effect on the short term social and 762 discriminative memories (STM) and of recent long term memory (LTM), and on 763 markers of oxidative stress in the hippocampus (target structure in which the 764 neuroinflamation process occurred) and in the prefrontal cortex of mice (control 765 structure).

766 Regarding the biochemical tests, we observed that the muscular strength 767 exercise was able to promote increase of the activity of the antioxidant enzyme 768 CAT in the hippocampus in mice, in which there was induction of local 769 neuroinflammation in the CA1 hippocampal region, both regarding the exercised 770 control groups, as well as regarding the group that had also induced local 771 neuroinfammation, but which did not suffer the previous protocol of strength 772 exercise. In the prefrontal cortex, it was verified that the muscular strength 
773 exercise reduced the activity of the enzyme CAT in such brain region, compared 774 with the mice that were not exercised, except for the reduction in the activity of 775 CAT in the exercised group.

776 In the object recognition test, we discovered that the prophylactic strength 777 exercise was able to revert the amnesia deficit caused by the LPS regarding both 778 types of memory, STM and LTM. Regarding social memory, the exercise 779 prevented amnesia deficit in LTM.

The oxidative stress, the mitochondrial dysfunction and the hyperactivity 781 of microglia are key-processes involved in the neuroinflammatory physio782 pathology of different brain diseases [8]. As a response to a lesion, during 783 maximum activation of microglia, an increase in its cell metabolism occurs, as 784 well as synthesis of pro-inflammation factors, in order to eliminate the stressful 785 stimulation and maintain the tissue homeostasis. However, associated to an 786 increase in the cell metabolism, it also occurs the production of different reactive 787 species of oxygen, such as super-oxide radicals $\left(\mathrm{O}_{2}^{-}\right)$, hydroxyl $\left(\mathrm{OH}^{-}\right)$and, yet, 788 hydrogen peroxide $\left(\mathrm{H}_{2} \mathrm{O}_{2}\right)$. The excessive formation of such free radicals may lead to DNA alterations 790 and lipidic peroxidation, key-factors in the acceleration of the neuroinflammation 791 process related to age and to the development of neurodegenerative diseases 792 [10].

The hippocampus is highly vulnerable to an increase in the formation of 794 free radicals due to its reduced capacity of keeping the redox homeostasis [32]. 795 Since the hippocampus is involved in certain ways of learning and memory 796 consolidation [33], a chronic local formation of reactive species of oxygen may 
797

798

cause impairment of the mnemonic functions. Therefore, the maintenance of a redox normal state in the hippocampal microglia is important for the prevention of a cognitive decline [32].

To protect itself from these reactive species, the microglia reacts through anti-oxidant mechanisms of defense, and one of such mechanisms acts through specialized enzymes, such as glutathione-peroxidase (GSH-Px), superoxidedismutase (SOD) and CAT [34]. The second line of defense has the function of repairing a possible damage which has occurred in the cell, being constituted by substances such as glutathione-redutase (GSH-Rd), GSH-Px, among others.

Physical exercise has shown to be promising in attenuation of an oxidative stress. During the practice of the physical exercise, there is an increase of around $40 \%$ to $70 \%$ in the brain blood flow to supply the metabolic demand of oxygen [32]. Such increase in the consumption of oxygen results in the microglia in an increase in the synthesis of reactive species of oxygen as a response to the intensity of the exercise.

Therefore, it is believed that other factors which increase the production of ROS in the microglia, such as infections, mechanical traumas and ischemia, will be more easily reduced by endogenous factors of protection in individuals who practice physical exercise regularly, either by the positive regulation of antioxidant enzymes and/or by increase in the number of mitochondria which allow lower levels of respiratory activity for the same degree of ATP generation [35]. The studies of $[36,37]$ show that different factors in the mitochondria are modulated by redox alterations associated to the exercise, through the activation of the transcriptional coactivator peroxisome proliferator-activated receptor- $\gamma$ 


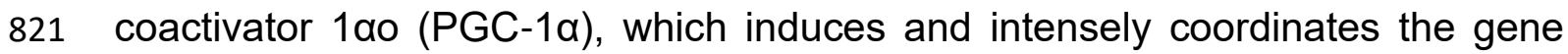
822 expression that stimulates the oxidative mitochondrial metabolism and may 823 reduce in diminishing of the oxidative stress.

824 In fact, we observed that the animals that received the intra-hippocampal 825 injection of LPS in the CA1 region of the hippocampus obtained an increase in 826 the hippocampal concentration of GSH. Such increase that may have occurred 827 as a local homeostasis answer that increased the production of such anti-oxidant 828 as defense against the increase of free radicals unleashed by the inflammatory 829 response. In addition, the increase of the concentration of GSH was the single 830 antioxidant answer that we detected in this study, once the activities of the 831 enzymes here evaluated were not altered in the hippocampus due to the local 832 inflammation caused by the infusion of the LPS.

833 However, the muscular strength exercise showed to have positive 834 influence on the activity of the anti-oxidant enzymes CAT in the animals which 835 received LPS after having practiced the muscular strength exercise. Thus, once 836 again, this did not have effect on the activity of the enzymes MPO, SOD and GST. 837 Regarding the concentration of $\mathrm{GSH}$, the muscular strength exercise caused a 838 diminishing of such concentration in the hippocampus of mice which suffered the 839 induction of hippocampal neuroinflammation, regarding the group of mice which 840 also suffered such induction, but were not previously exercised. This may indicate 841 that, in some way, consequent to the previous performance of the strength 842 exercise, there has not been, after the induction of the hippocampal 843 neuroinflammation, an oxidative unbalance strong enough to result in higher 844 production of GSH to supply a higher demand for this antioxidant (hypothesis of 
845 diminishing the production of $\mathrm{GSH}$ ), or if there was a higher consumption of 846 additional GSH produced precisely to diminish the concentration of the ROE 847 resulting from the neuroinflammatory process (hypothesis of increased 848 consumption of GSH). The increase in the activity of CAT and the diminishing of 849 the mnemonic deficit observed in some behavioral tasks link with such suggested 850 explanation.

851 We also verified that the strength exercise reduced the activity of the CAT 852 in the prefrontal cortex, which by itself is an interesting result. Such result, 853 perhaps, signs that the routine of muscular strength exercise diminishes the 854 demand for the constitutive action of CAT in such brain region, which indicates 855 that there would be a lower generation of ROE in the mice submitted to a 856 muscular strength exercise routine. However, such reduction did not occur in 857 mice that suffered induction of local neuroinflammation in the hippocampal 858 region. In such mice, the activity of CAT was kept at the same level whether the 859 mice had or had not been submitted to the prophylactic protocol of muscular 860 strength exercise, which was a similar level to the groups NAIVE and SAL from 861 the non-exercised animals. This is another interesting result, highlighting that an 862 inflammatory process triggered in a brain region (hippocampus) rebounds in 863 other regions, in this case, on the modulation of the effect of the routine of 864 muscular strength physical exercise on the activity of CAT. Here, we may 865 consider that the hippocampal neuroinflammation rebounded in several brain 866 regions, including the prefrontal cortex, causing in increase in the activity of CAT, 867 which would counterbalance the reduction of the CAT activity induced by the 868 strength exercise. However, if that was the case, we should have noticed an 
869 increase in the activity of CAT in the prefrontal CAT in non-exercised mice that 870 suffered local neuroinflammation in the hippocampus, which was not verified in 871 our results. Therefore, what we had here was that, in some way, the 872 neuroinflammation induction in the hippocampus provokes an increase of the 873 activity of CAT in the prefrontal cortex when it is already reduced regarding the 874 default levels (from the non-exercised groups), being such reduction attributed to 875 the previous routine of muscular strength exercise. With the purpose of maintaining the basal levels of ROS and/or nitrogen,

877 the enzymactic and non-enzymactic antioxidants perform different functions. The 878 enzyme SOD catalyzes the desmutation of $\mathrm{O}_{2}^{-}$converting it into $\mathrm{H}_{2} \mathrm{O}_{2}$, soon the 879 enzyme CAT catalyzes the conversion of hydrogen peroxide into water and 880 molecular oxygen, which is a function also performed by the enzyme glutathione881 peroxidase [38, 39]. Regarding the enzyme GST, it acts helping the conjugation 882 of eletrofilic substrates to glugathione (GSH) $[40,41]$. Therefore, we believe that 883 the increase in the activity of the enzymes CAT in animals that performed 884 muscular strength exercise and after suffered the induction of hippocampal 885 neuroinflammation has been given by an increase in the production of hydrogen 886 peroxide, aiming at reducing it to water and oxygen and maintaining a 887 neuroprotector effect, thus, despite not being a free radical, by the absence of 888 unpaired electrons in the last layer, $\mathrm{H}_{2} \mathrm{O}_{2}$ is an extremely deleterious $\mathrm{ROE}$, 889 capable of crossing lipid layers and reacting with transition metals and some 890 hemoproteins. It can also induce chromosomal alterations, by bursting the DNA 891 column and, in the absence of catalysts, oxidizing sulfhydryl composites. 
The overproduction of $\mathrm{H}_{2} \mathrm{O}_{2}$, in addition to enabling the induction of the

893 increase in the activity of the enzymes CAT as a protection factor, can also 894 promote the inactivation of the enzymes SOD, which was verified in different 895 studies that suggest that high concentrations of $\mathrm{H}_{2} \mathrm{O}_{2}$ inactivated the enzyme 896 SOD [42, 43], corroborating our result, in which there were no statistical 897 differences in the quantification of the enzyme SOD among the groups. with the results from the behavioral analysis evaluated in our study, since with 900 the increase/adaptation of the antioxidant enzyme given by the muscular strength 901 exercise, we verified a neuroprotective effect against the mnemonic deficits in 902 animals which suffered the intra-hippocampal infusion of LPS.

903 Corroborating with our study, it was investigated in [44] the effect of 904 strength exercise in mice after an induced neuroinflammation by monosodium 905 glutamate. The animals received monosodium glutamate in the dose of $4 \mathrm{~g} / \mathrm{kg}$, on 906 the first day of life until the 10th day. After 60 days after the day of birth, the 907 animals initiated a strength exercise protocol for 7 weeks and, posteriorly, the 908 object recognition test was performed. The authors verified that the strength 909 exercise had effect in diminishing the deficit of discriminative memory.

910 According to [45], beneficial effects from the muscular strength exercise 911 were also related in the deficit of discriminative memory. The authors observed 912 that after eight weeks of muscular strength training, the animals received an 913 intracerebralventricular injection of the peptide $A \beta_{25-35}$ and practiced exercise 914 presented a significant improvement when compared to the control group 915 regarding deficit of the discriminative memory. 
According to [46], the neuroprotective effects of three models of non-

917 pharmaceutical intervention, environmental enrichment, muscular strength

918

919

920

921

922

923

924

925

926 discriminative memory deficits. Still, when the studies here quoted showed the of

927 muscular strength exercise as a form of treatment, our study showed its

928

929

930

931

932

933

934

935

936 animals that received intra-hippocampal LPS in the group of animals that

937 practiced strength exercise may have contributed for the improvement of the

938 performance of such animals in the evaluation of the retention of discriminative

939 and social retention, possibly due to the diminishing of the ROE. 


\section{CONCLUSION}

In conclusion, the present study reinforces evidences that the muscular

943 strength exercise represents a useful prophylactic approach for the prevention of

944 behavioral deficiencies and of oxidative stress associated to neuroinflammation, 945 as it was induced in this study by LPS. We showed the beneficial effects of 946 strength exercise in animals that suffered local hippocampal neuroinflammation, 947 having avoided the mnemonic deficit in tasks of discriminative and social 948 memory, promoting the increase of the activity of the enzyme CAT and the 949 diminishing of the concentration of GSH (whether due to lower demand of GSH 950 or by inducing its higher use) in the hippocampus.

\section{DECLARATIONS}

\section{Ethics approval}

955 All the experiments were conducted in accordance with the principles of 956 laboratory animal care (National Research Council Committee for the Update of 957 the Guide, 2011) and were approved by the Institutional Animal Care and Use 958 Committee of the Local Institution (CEUA/UNIPAMPA) - Protocol \#046/2017. 959

960 Consent for publication

961 Not applicable. 
965 The datasets used and/or analysed during the current study are available from

966 the corresponding author on reasonable request.

967

968 Competing interests

969 The authors declare that they have no competing interests.

970

971 Funding

972 This study was financed in part by the Conselho Nacional de Desenvolvimento

973 Científico e Tecnológico (CNPq).

974

975 Authors' contributions

976 EG was responsible for the acquisition of all research data,Writing- Original draft

977 preparation and Writing- Reviewing and Editing; GCM, LBS, LFL, KRL, GSC,

978 BHSN, SSP contributed largely to the research data acquisition; JSB contributed

979 revising the manuscript critically for important intellectual content and PBMC

980 supervised all research steps and contributed with Conception and design of

981 study; WCS performed the statistical analyzes, contributed with the design of

982 study,Writing- Original draft preparation and Writing- Reviewing and Editing. All

983 authors approved the version of the manuscript to be published.

984

985 Acknowledgments 
986 This study was financed in part by the Conselho Nacional de Desenvolvimento 987 Científico e Tecnológico (CNPq) and Universidade Federal do Rio Grande do Sul 988 (UFRGS).

989

990 REFERENCES

991 [1] Niranjan R. Recent advances in the mechanisms of neuroinflammation and 992 their roles in neurodegeneration. Neurochemistry International. 2018, 120:13-20.

993 [2] Schain M, Kreisl WC. Neuroinflammation in Neurodegenerative Disorders994 a Review. Current Neurology And Neuroscience Reports. 2017, 17.

995 [3] Seo Dae-yun et al. Exercise and Neuroinflammation in Health and 996 Disease. International Neurourology Journal. 2019, 23:82-92.

997 [4] Shabab T et al. Neuroinflammation pathways: a general review. International 998 Journal of Neuroscience. 2016, 126:624-633.

999 [5] Cai L et al. Antidepressant-like effects of penta-acetyl geniposide in chronic 1000 unpredictable mild stress-induced depression rat model: Involvement of inhibiting 1001 neuroinflammation in prefrontal cortex and regulating hypothalamic1002 pituitaryadrenal axis. International Immunopharmacology. 2020, 80:106182.

1003 [6] Hou L et al. NADPH oxidase and neurodegenerative diseases: mechanisms 1004 and therapy. Antioxidants \& Redox Signaling. 2020, 33:374-393.

1005 [7] Binvignat, O, Olloquequi, J. Excitotoxicity as a Target against 1006 Neurodegenerative Processes. Current Pharmaceutical Design. 2020, 26:125110071262. 
1008 [8] d'avila JC et al. Age-related cognitive impairment is associated with long-term 1009 neuroinflammation and oxidative stress in a mouse model of episodic systemic 1010 inflammation. Journal of Neuroinflammation. 2018, 15.

1011 [9] Sung LL. Neuroinflammation and Neurogenesis in Alzheimer's Disease and 1012 Potential Therapeutic Approaches. International Journal of Molecular Sciences. $10132020,21: 701-723$.

1014 [10] Buendia I et al. Nrf2-ARE pathway: An emerging target against oxidative 1015 stress and neuroinflammation in neurodegenerative diseases. Pharmacology \& 1016 Therapeutics. 2016, 157:84-104.

1017 [11] Pérez-Domínguez M, Tovar-Y-Romo LB, Zepeda A. Neuroinflammation and 1018 physical exercise as modulators of adult hippocampal neural precursor cell 1019 behavior. Reviews In The Neurosciences. 2017, 29:1-20.

1020 [12] Knaepen K et al. Neuroplasticity - Exercise-Induced Response of Peripheral 1021 Brain-Derived Neurotrophic Factor. Sports Medicine. 2010, 40:765-801.

1022 [13] Stranahan AM, Khalil D, Gould E. Social isolation delays the positive effects 1023 of running on adult neurogenesis. Nature Neuroscience. 2006, 9:526-533.

1024 [14] Vaynman S, Gomez-Pinilla F. License to Run: Exercise Impacts Functional 1025 Plasticity in the Intact and Injured Central Nervous System by Using 1026 Neurotrophins. Neurorehabilitation and Neural Repair. 2005, 19:282-295.

1027 [15] Mokhtari-Zaer A et al. Treadmill exercise restores memory and hippocampal 1028 synaptic plasticity impairments in ovalbumin-sensitized juvenile rats: Involvement 1029 of brain-derived neurotrophic factor (BDNF). Neurochemistry International. 2020, 1030 135:104691. 
1031 [16] Ding $Q$ et al. Insulin-like growth factor I interfaces with brain-derived 1032 neurotrophic factor-mediated synaptic plasticity to modulate aspects of exercise1033 induced cognitive function. Neuroscience. 2006, 140:823-833.

1034 [17] Schimidt $\mathrm{HL}$ et al. Strength training and running elicit different 1035 neuroprotective outcomes in a $\beta$-amyloid peptide-mediated Alzheimer's disease 1036 model. Physiology \& Behavior. 2019, 206:206-212.

1037 [18] TORII $Y$ et al. Comparison of effects of botulinum toxin subtype A1 and A2 1038 using twitch tension assay and rat grip strength test. Toxicon, [s.I.] 2011, v. 57, n. 1039 1, p.93-99.

1040 [19] Lima KR et al. Noradrenergic and dopaminergic involvement in novelty 1041 modulation of aversive memory generalization of adult rats. Behavioural Brain 1042 Research. 2019, 371: 111991.

1043 [20] Vargas LS et al. One-single physical exercise session after object 1044 recognition learning promotes memory persistence through hippocampal 1045 noradrenergic mechanisms. Behavioural Brain Research. 2017, 329:120-126.

1046 [21] Kaidanovich-beilin $O$ et al. Assessment of Social Interaction 1047 Behaviors. Journal of Visualized Experiments 2011, 1-6.

1048 [22] Amoah SK. et al. Inhibitory effect of sesquiterpene lactones and the 1049 sesquiterpene alcohol aromadendrane-4 $\beta, 10 \alpha$-diol on memory impairment in a 1050 mouse model of Alzheimer. European Journal Of Pharmacology. 2015, 769:1951051202.

1052 [23] Pellow S. et al. Validation of open: closed arm entries in an elevated plus1053 maze as a measure of anxiety in the rat. Journal Of Neuroscience Methods. 1985, 1054 14:149-167. 
1055 [24] Tita B et al. Analgesic properties of Epilobium angustifolium, evaluated by 1056 the hot plate test and the writhing test. II Farmaco. 2011, 56:5-7.

1057 [25] Bradley PP, Priebat, DA, Christenses, RD. Measurement of Cutaneous 1058 Inflammation: Estimation of Neutrophil Content with an Enzyme Marker. J. Invest. 1059 Dermatol. 1982, 78: 206-209.

1060 [26] Sedlak J, Lindsay RH. Estimation of total prote in bound and nonprotein 1061 sulfhydril groups in tissues with Ellman's reagent. Anal. Biochem. 1968, 25: 1921062205.

1063 [27] Jiang ZY, Woollard ACS, Wolff SP. Lipid hydroperoxide measurement by 1064 oxidation of $\mathrm{Fe} 2+$ in the presence of xylenol orange. Comparison with the TBA 1065 assay and an iodometric method. Lipids. 1991, 26: 853-856.

1066 [28] Marklund S, Marklund G. Involvement of the Superoxide Anion Radical in the 1067 Autoxidation of Pyrogallol and a Convenient Assay for Superoxide Dismutase. 1068 Eur. J. Biochem. 1974, 47: 469-474.

1069 [29] Aebi H. Catalase. Methods Enzymol. 1984, 105: 121-126.

1070 [30] Habig WH. Pabst MJ. Jakoby WB. Glutathione S-transferases. The first 1071 enzymatic step in mercapturic acid formation. J. Biol. Chem. 1974, 249: 713010727139.

1073 [31] Izquierdo I, Medina JH. Memory formation - the sequence of biochemical 1074 events in the hippocampus and its connection to activity in other brain structures. 1075 Neurobiol. Learn. Mem. 1997, 68: 285-316.

1076 [32] Marosi K, et al. Long-term exercise treatment reduces oxidative stress in the 1077 hippocampus of aging rats. Neuroscience. 2012, 226:21-28. 
1078 [33] Morris RGM. Elements of a neurobiological theory of hippocampal function:

1079 the role of synaptic plasticity, synaptic tagging and schemas. European Journal 1080 of Neuroscience. 2006, 23:2829-2846.

1081 [34] Baumann CW et al. Age-induced oxidative stress: how does it influence 1082 skeletal muscle quantity and quality?. Journal Of Applied Physiology. 2016, 1083 121:1047-1052.

1084 [35] Sen CK et al. Skeletal muscle and liver glutathione homeostasis in response 1085 to training, exercise, and immobilization. Journal of Applied Physiology. 1992, 1086 73:1265-1272.

1087 [36] Radak Z et al. Oxygen Consumption and Usage During Physical Exercise: 1088 The Balance Between Oxidative Stress and ROS-Dependent Adaptive 1089 Signaling. Antioxidants \& Redox Signaling. 2013, 18:1208-1246.

1090 [37] Liu $\mathrm{Y}$ et al. Short-term resistance exercise inhibits neuroinflammation and 1091 attenuates neuropathological changes in 3xTg Alzheimer's disease mice. Journal 1092 of Neuroinflammation. 2020, 17.

1093 [38] Glorieux C, Calderon PB. Catalase, a remarkable enzyme: targeting the 1094 oldest antioxidant enzyme to find a new cancer treatment approach. Biological 1095 Chemistry. 2017, 398:1095-1108.

1096 [39] Tehrani HS, Moosavi-Movahedi AA. Catalase and its mysteries. Progress In 1097 Biophysics And Molecular Biology. 2018, 140:5-12.

1098 [40] Strange RC et al. Glutathione-S-transferase family of enzymes. Mutation 1099 Research. Fundamental And Molecular Mechanisms Of Mutagenesis. 2001, $1100 \quad 482: 21-26$. 
1101 [41] Özaslan MS et al. Evaluation of chalcones as inhibitors of glutathione S1102 transferase. Journal of Biochemical And Molecular Toxicology. 2018, 32:1-3.

1103 [42] Salo DC et al. Superoxide dismutase is preferentially degraded by a 1104 proteolytic system from red blood cells following oxidative modification by 1105 hydrogen peroxide. Free Radical Biology And Medicine. 1998, 5:335-339.

1106 [43] Sampson JB, Beckman JS. Hydrogen Peroxide Damages the Zinc-Binding 1107 Site of Zinc-Deficient Cu, Zn Superoxide Dismutase. Archives Of Biochemistry 1108 And Biophysics. 2001, 392:8-13.

1109 [44] Araujo PCO et al. Resistance exercise reduces memory impairment 1110 inducedby monosodium glutamate in male and female rats. Experimental 1111 Physiology. 2017, 102:845-853.

1112 [45] Farzi MA et al. Exercise Improves Recognition Memory and

1113 Acetylcholinesterase Activity in the Beta Amyloid-Induced Rat Model of

1114 Alzheimer's Disease. Annals Of Neurosciences. 2018, 25:121-125.

1115 [46] Quines CB et al. Resistance training restores metabolic alterations induced 1116 by monosodium glutamate in a sex-dependent manner in male and female rats. 1117 Journal Of Cellular Biochemistry. 2019, 8:1-7. 